\title{
Potential of Higher-Order Moments and Slopes of the Radar Doppler Spectrum for Retrieving Microphysical and Kinematic Properties of Arctic Ice Clouds $\mathscr{A}$
}

\author{
MAXIMILIAN MAAHN ${ }^{\mathrm{a}}$ AND ULRICH LÖHNERT \\ Institute for Geophysics and Meteorology, University of Cologne, Cologne, Germany
}

(Manuscript received 22 December 2015, in final form 2 September 2016)

\begin{abstract}
Retrievals of ice-cloud properties from cloud-radar observations are challenging because the retrieval methods are typically underdetermined. Here, the authors investigate whether additional information can be obtained from higher-order moments and the slopes of the radar Doppler spectrum such as skewness and kurtosis as well as the slopes of the Doppler peak. To estimate quantitatively the additional information content, a generalized Bayesian retrieval framework that is based on optimal estimation is developed. Real and synthetic cloud-radar observations of the Indirect and Semi-Direct Aerosol Campaign (ISDAC) dataset obtained around Barrow, Alaska, are used in this study. The state vector consists of the microphysical (particle-size distribution, mass-size relation, and cross section-area relation) and kinematic (vertical wind and turbulence) quantities required to forward model the moments and slopes of the radar Doppler spectrum. It is found that, for a single radar frequency, more information can be retrieved when including higher-order moments and slopes than when using only reflectivity and mean Doppler velocity but two radar frequencies. When using all moments and slopes with two or even three frequencies, the uncertainties of all state variables, including the mass-size relation, can be considerably reduced with respect to the prior knowledge.
\end{abstract}

\section{Introduction}

Ice clouds play a key role in the atmosphere of Earth, but in situ observations of ice clouds are difficult to make and thus are sparse. Remote sensing instruments have the potential to fill this gap because they can provide observations at high temporal and spatial resolutions. Among remote sensing instruments, ground-based vertically pointing Doppler cloud radars are the only instruments that can penetrate optically thick ice clouds and provide measurements of the fall velocity of hydrometeors. However, the observables of cloud radars are only indirectly linked to cloud and precipitation properties. The required transfer functions are not uniquely defined, resulting in substantial uncertainties in radar-based ice-cloud retrievals.

\footnotetext{
O) Supplemental information related to this paper is available at the Journals Online website: http://dx.doi.org/10.1175/ JAMC-D-16-0020.s1.

${ }^{\text {a }}$ Current affiliation: Cooperative Institute for Research in Environmental Sciences, University of Colorado Boulder, and NOAA/ Earth System Research Laboratory, Boulder, Colorado.
}

Corresponding author e-mail: Maximilian Maahn, maximilian. maahn@colorado.edu
Different strategies have been used to increase the information content of radar observations: some studies suggested the use of radar-microwave radiometer combinations (Grecu and Olson 2008; Posselt and Mace 2014), radar-lidar combinations (Intrieri et al. 1993; Delanoë and Hogan 2008), or multiple radars operating at two (Hogan et al. 2000; Szyrmer and Zawadzki 2014) or three frequencies (Sekelsky et al. 1999; Kneifel et al. 2011). For single-instrument retrievals, some studies proposed to exploit not only equivalent radar reflectivity factor $Z_{e}$ but also mean Doppler velocity $W$ (Matrosov et al. 2002; Szyrmer et al. 2012) and Doppler spectrum width $\sigma$ (Mace et al. 2002; Deng and Mace 2006) of zenith-pointing radars. Also, the use of the full radar Doppler spectrum has been suggested (Verlinde et al. 2013).

Our research addresses the point of increasing the information content of observations from ice-cloud radars by studying whether additional information can be obtained from the higher-order moments and the slopes of the radar Doppler spectrum. For this, not only are $Z_{e}, W$, and $\sigma$ studied but also skewness $\gamma$ and kurtosis $\kappa$. In addition to the higher-order moments, the left slope $S_{l}$ and the right slope $S_{r}$ of the radar Doppler maximum peak are also investigated. While $\sigma, \gamma$, and $\kappa$ have the advantage 
that they are not influenced by radar calibration, they are strongly influenced by turbulence. Therefore, the retrieval implemented in this study provides not only microphysical properties - such as particle-size distribution, mass-size relation, and cross section-area relationbut also kinematic variables, such as vertical air motion and turbulent spectral broadening.

Lack of information about the mass-size relation of hydrometeors is one major reason for the uncertainty of most radar retrievals. A few studies solve this problem by including particle mass (or density) into the retrieval (e.g., Posselt and Mace 2014; Szyrmer and Zawadzki 2014). A retrieval providing microphysical parameters including the mass-size relation as well as kinematic properties, together with the corresponding uncertainties, has, to the authors' knowledge, not yet been developed. The same is true for ice-cloud retrievals using higherorder moments even though there are studies using $\gamma$ and $\kappa$ for detection of drizzle onset (e.g., Kollias et al. 2011) and to locate supercooled liquid water (e.g., Luke et al. 2010). Maahn et al. (2015) found higher-order radar moments to be useful for evaluating ice-cloud parameterizations using observations from the Indirect and Semi-Direct Aerosol Campaign (ISDAC; McFarquhar et al. 2011), which took place in 2008 in the environs of the Atmospheric Radiation Measurement Program North Slope of Alaska (NSA) site in Barrow, Alaska. The dataset and the parameterizations recommended by Maahn et al. (2015) are the basis for this study.

To study the information content of higher-order moments and slopes qualitatively, response functions to ice-cloud parameters are assessed. For a quantitative analysis, the number of independent information pieces (total degrees of freedom for signal $D_{f}$ ) are estimated using a retrieval that is based on optimal-estimation theory (Rodgers 2000). Optimal estimation is widely used in atmospheric remote sensing, in particular for passive and active microwave applications (e.g., Löhnert et al. 2004; Steinke et al. 2014).

The radar simulator of the Passive and Active Microwave Radiative Transfer (PAMTRA) model (Maahn 2015) is utilized as the forward operator. As input, both real and synthetic radar observations from the ISDAC campaign are used. The retrieval output is composed of variables that describe the particle-size distribution $N(D)$, the mass-size relation $m(D)$, and the cross section-area relation $A(D)$ and kinematic variables that are related to turbulence and wind. For this, the a priori dataset and the parameterizations recommended by Maahn et al. (2015) are used. They used maximum particle dimension as the size descriptor, which is adopted here for consistency. Optimal estimation provides not only a retrieved state but also its uncertainty, assuming a Gaussian uncertainty distribution. Therefore, the analysis of retrieval results is not limited to bias but also covers uncertainties. The retrieval results are further analyzed in terms of the use of additional radar frequencies, the impact of calibration offset, measurement uncertainty, and prior knowledge of kinematic variables.

The datasets used, the forward model, and the optimalestimation retrieval algorithm are described in section 2 . The response functions of radar moments and slopes to microphysical parameters are discussed in section 3. In section 4 , the retrieval results are investigated, and the impact of modifications to the retrieval is analyzed in section 5. The findings are discussed and concluding remarks are given in section 6 .

\section{Setup}

In this section, the datasets and methods used in this study are introduced. This includes the definition of the state vector $\mathbf{x}$ and the measurement vector $\mathbf{y}$ and how they are connected by optimal estimation.

\section{a. Dataset}

All data used in this study were obtained during the ISDAC campaign (McFarquhar et al. 2011; Jackson et al. 2012), which took place in 2008 at the NSA site in Barrow and covered mostly stratocumulus ice and mixed-phase clouds. The ISDAC dataset consists of in situ cloud measurements by the Convair aircraft (McFarquhar and Jackson 2012) as well as groundbased spectral radar observations by the millimeterwavelength cloud radar (MMCR) operating at a frequency of $34.86 \mathrm{GHz}$ (Moran et al. 1998), which are available on request (Bharadwaj and Johnson 2003). Supercooled liquid water (SCLW) can be visible in the radar Doppler spectrum at high concentrations (Luke et al. 2010), which could bias the analysis. Therefore, only aircraft observations in the vicinity of the NSA site that contain less than $0.01 \mathrm{~g} \mathrm{~m}^{-3}$ of SCLW as measured by the "King" probe are used for this study. Even though such low SCLW values are, in the presence of cloud ice, usually not visible in the radar spectrum, rimed ice particles are probably still included in the dataset. Most of the observed clouds were precipitating, but aircraft observations were usually above cloud base. In the following, the quantities derived from the ISDAC dataset are briefly introduced. See Maahn et al. (2015) for a detailed discussion about why the following methods are used to parameterize the ISDAC dataset.

Particle mass is expressed by a normalized power law as proposed by Szyrmer et al. (2012): 


$$
m(D)=\alpha C_{0}\left(D / D^{*}\right)^{b}
$$

where $D^{*}$ and $C_{0}$ describe a reference particle size and mass, respectively, and $\alpha$ is a dimensionless factor. In accordance with Szyrmer et al. (2012) and Maahn et al. (2015), $D^{*}$ and $C_{0}$ are set to $1.2 \times 10^{-3} \mathrm{~m}$ and $3 \times 10^{-8} \mathrm{~kg}$, respectively. In comparison with the common power-law formulation of the mass-size relation $m(D)=a D^{b}$, the normalized version is more robust and has less correlation between the parameters [cf. with Wood et al. (2014)] but still has two degrees of freedom. Because the coefficients $\alpha$ and $b$ have not been measured during ISDAC, they are retrieved as a closure from a combination of ISDAC airborne measurements and groundbased radar observations of the MMCR as shown in Maahn et al. (2015). Factor $\alpha$ can be transformed to the $a$ parameter of the more commonly used power-law mass-size relation with $a=\alpha C_{0} /\left(D^{*}\right)^{b}$. Note that the $b$ exponent value of 2.2 found by Maahn et al. (2015) is closer to the values found by, for example, Heymsfield et al. (2010) (2.1-2.2) and Wood et al. (2014) (2.1) than to the value of 1.9 found by Brown and Francis (1995) that is frequently used for remote sensing.

The particle-size distribution $N(D)$ is estimated from the aircraft observations using the a normalized gamma distribution by Testud et al. (2001) and Delanoë et al. (2005). Instead of the equivalent melted diameter, the maximum particle dimension $D$ is used as particle-size descriptor as introduced in Maahn et al. (2015):

$$
\begin{aligned}
N(D)= & N_{0}^{*} \frac{(b+\mu+1)^{b+\mu+1} \Gamma(b+1)}{\Gamma(b+\mu+1)(b+1)^{b+1}}\left(\frac{D}{D_{m}}\right)^{\mu} \\
& \times \exp \left[-(b+\mu+1) D / D_{m}\right],
\end{aligned}
$$

where $N_{0}^{*}$ is the intercept parameter, $D_{m}$ is the massweighted scaling parameter, $\mu$ describes the shape of the distribution, $\Gamma$ is the gamma function, and $b$ is the exponent of the mass-size relation. Note that in the following, $\mu$ is replaced by $\mu^{*}=b+\mu+1$ to increase the numerical stability of the gamma function when using it within a retrieval. The coefficients $N_{0}^{*}$ and $D_{m}$ are derived directly from the moments of the particle distributions measured by the cloud probes; $\mu^{*}$ is obtained by a least squares fit to the measured distribution. Because rare, large particles cannot be sufficiently sampled with in situ probes, $N(D)$ is extrapolated to the largest size class $(12.8 \mathrm{~mm})$ of the in situ probes as discussed in Maahn et al. (2015).

The projected area $A(D)$ is required for estimation of the quiet-air fall velocity. A power law is used to describe the observations:

$$
A(D)=c D^{d}
$$

with prefactor $c$ and exponent $d$. Maahn et al. (2015) found that an additional noise vector has to be applied to the area-size relation allowing for a deviation of $A(D)$ from the power law so as to match the radar moments observed by the MMCR. However, a random term cannot be handled properly by the optimal-estimation framework, and the corresponding error is treated as forward-model error instead (see section $2 \mathrm{~h}$ ). Note that this random vector is, however, used when creating synthetic radar observations.

For kinematic effects, vertical air motion $w$ (resulting in a shift of the Doppler peak) and kinematic broadening $\sigma_{k}$ of the Doppler peak are considered. As discussed in Maahn et al. (2015), $\sigma_{k}$ depends mainly on the horizontal wind $u$, the turbulence expressed by the eddy dissipation rate $\varepsilon$, the radar integration time, and the radar beamwidth. The $u$ and $w$ are obtained from aircraft measurements, whereby $w$ is offset corrected as suggested by Gultepe et al. (1990). Even though the Convair aircraft was equipped with a Tropospheric Airborne Meteorological Data Report (TAMDAR) instrument (Moninger et al. 2010) that was capable of measuring turbulence, during ISDAC $\varepsilon$ was mostly below the detection threshold of $10^{-3} \mathrm{~m}^{2} \mathrm{~s}^{-3}$ of the TAMDAR instrument. As a consequence, the TAMDAR instrument only provides an upper bound of $\varepsilon$; for ISDAC, Maahn et al. (2015) estimated $\varepsilon$ to be $10^{-6} \mathrm{~m}^{2} \mathrm{~s}^{-3}$ on the basis of the comparison of in situ aircraft and ground-based radar observations.

\section{b. Forward model}

To simulate the full radar spectrum $s$ and the corresponding moments and slopes on the basis of the provided in situ hydrometeor profiles, the PAMTRA model is used (Maahn 2015). Different from the method in Maahn et al. (2015), the self-similar Rayleigh-Gans (SSRG) approximation (Hogan and Westbrook 2014) is used here instead of the soft spheroid $\mathbf{T}$-matrix approach (Mishchenko 2000) to estimate the ice-particle scattering properties. The approximation is orders of magnitude faster than the spheroidal T-matrix approach and is applicable to any particle type, thereby providing more robust results for particles that are larger than the radar wavelength $\lambda$ (see the online supplemental material). SSRG depends on $D, m$, and aspect ratio AR, which was fixed at 0.6 (assuming horizontal alignment) in accordance with Hogan et al. (2012) and because no dependence of higher-order moments and slopes to $A R$ was found by Maahn et al. (2015). In contrast to the soft spheroid T-matrix approach, the refractive index of pure 
ice (Warren and Brandt 2008) is used for SSRG. The quiet-air particle fall velocity of the hydrometeors is estimated using the method of Heymsfield and Westbrook (2010), which depends on $D, m(D), A(D)$, and air density. The resulting quiet-air radar Doppler spectrum is convolved with a Gaussian distribution with standard deviation $\sigma_{k}$ to account for kinematic broadening and is shifted by $w$ to account for vertical air motion. Last, noise is applied in accordance with the MMCR specifications.

In this study, the PAMTRA forward simulator is configured in accordance with the technical specifications of the MMCR that was operating during ISDAC. It operated at a frequency of $34.86 \mathrm{GHz}$ with Doppler velocity resolution of $4.1 \mathrm{~cm}$, a full-width beamwidth of $0.31^{\circ}$, and a noise level of $-33.25 \mathrm{dBz}$ [as defined by Smith (2010)] at 1-km height (Moran et al. 1998; Bharadwaj and Johnson 2003). As attenuation is expected to be negligible for snow and ice at Ka band (Matrosov 2007), attenuation is not considered here.

\section{c. Radar observables}

The radar Doppler spectrum $\hat{s}$ (SI units: $\mathrm{m}^{2} \mathrm{~m}^{-3}$ ) is converted to $s\left(\mathrm{~mm}^{6} \mathrm{~m}^{-3}\right)$ with

$$
s=10^{18} \frac{\lambda^{4}}{\left|K_{w}\right|^{2} \pi^{5}} \hat{s},
$$

where $\lambda$ is the radar wavelength and $\left|K_{w}\right|^{2}$ is the dielectric factor for water. It is convention to fix $\left|K_{w}\right|^{2}$ to 0.93 for the Ka band. Note that neither $s$ nor $\hat{s}$ is normalized with the Doppler resolution $\Delta v$.

With this definition, the equivalent radar reflectivity factor $Z_{e}$ can be obtained in decibels with respect to a reference level of $1 \mathrm{~mm}^{6} \mathrm{~m}^{-3}(\mathrm{dBz}$; Smith 2010) with

$$
Z_{e}=10 \log _{10} z_{e},
$$

where

$$
z_{e}=\sum_{i} s(i)
$$

The sum is taken over all bins $i$ of the Doppler spectrum that belong to the most significant peak. For consistency, PAMTRA's peak-recognition scheme is applied to both the MMCR Doppler spectra (Bharadwaj and Johnson 2003) and the synthetic spectra of PAMTRA's radar simulator, because moments and particularly slopes can depend on the method used for Doppler peak definition and recognition.

The mean Doppler velocity $W$ (first moment) is defined as

$$
W=\frac{\sum_{i} s(i) v(i)}{z_{e}},
$$

where $v(i)$ is the Doppler velocity of bin $i$. In the absence of vertical air motion, $W$ is equal to the reflectivityweighted mean particle fall velocity. There is no common convention for the sign of $W$; in this study positive $W$ refers to particles falling toward the ground.

The width of the spectrum is given by the standard deviation of the Doppler spectrum, called the Doppler spectrum width $\sigma$ (second central moment):

$$
\sigma=\left\{\frac{\sum_{i} s(i)[v(i)-W]^{2}}{z_{e}}\right\}^{1 / 2} .
$$

Width $\sigma$ depends not only on the particle-size distribution and quiet-air particle fall velocities but also on kinematic broadening by wind shear and turbulence occurring within the volume observed by the radar.

Skewness $\gamma$, the third central moment, is given by

$$
\gamma=\frac{\sum_{i} s(i)[v(i)-W]^{3}}{z_{e} \sigma^{3}}
$$

and describes whether the peak is skewed to the left $(\gamma<0)$ or right $(\gamma>0)$.

Kurtosis $\kappa$, the fourth central moment, defined as

$$
\kappa=\frac{\sum_{i} s(i)[v(i)-W]^{4}}{z_{e} \sigma^{4}},
$$

is a measure of the shape of the peak. A $\kappa$ equal to 3 indicates a Gaussian shape, smaller values of $\kappa$ indicate a more round peak, and spectra with a more pointed peak have $\kappa$ values larger than 3 .

In addition to the moments, the Doppler spectrum can also be described by the left slope $S_{l}$ and the right slope $S_{r}$ (Kollias et al. 2007) of the peak:

$$
\begin{aligned}
& S_{l}=10 \frac{\log _{10} N_{v}-\log _{10} s\left(i_{p}\right)}{v\left(i_{l}\right)-v\left(i_{p}\right)} \text { and } \\
& S_{r}=10 \frac{\log _{10} N_{v}-\log _{10} s\left(i_{p}\right)}{v\left(i_{r}\right)-v\left(i_{p}\right)}
\end{aligned}
$$

where $N_{v}$ is the mean spectral noise level and $i_{l}, i_{r}$, and $i_{p}$ are the indices of the leftmost, rightmost, and maximum bin of the Doppler peak, respectively. Note also that the sign of $\gamma$ and the definitions of $S_{l}$ and $S_{r}$ depend on the convention used for the sign of $\mathrm{W}$. The slopes, in 
contrast to the other moments are derived from the spectrum in logarithmic units. As a consequence, they are particularly sensitive to the tails of the peak. Slope $S_{l}$ depends on the particles with slow fall velocity; $S_{r}$ is governed by the fast-falling particles. In this study, the lower moments $Z_{e}$ and $W$ are collectively referred to as "LM" in contrast to $\sigma, \gamma, \kappa, S_{l}$, and $S_{r}$, which are referred to as higher moments, or "HM" (even though the slopes are not technically moments). All moments and slopes taken together are referred to as "AM."

\section{d. Optimal estimation}

Optimal estimation (Rodgers 2000) is a simplified Bayesian retrieval technique that is based on a Gaussian statistical model that combines the observation vector $\mathbf{y}$ of length $M$ with prior information to estimate the state vector $\mathbf{x}$ of dimension $N$. Optimal estimation requires that the forward operator $P$ be moderately linear and that the probability density distributions of state vector $\mathbf{x}$ and observation vector $\mathbf{y}$ follow a Gaussian shape. Then, the optimal solution of $\mathbf{x}$ can be found by an iterative procedure in which the updated state vector $\mathbf{x}_{i+1}$ is obtained as

$$
\begin{aligned}
\mathbf{x}_{i+1}= & \mathbf{x}_{a}+\left(\gamma_{i} \mathbf{S}_{a}^{-1}+\mathbf{K}_{i}^{\mathrm{T}} \mathbf{S}_{e}^{-1} \mathbf{K}_{i}\right)^{-1} \mathbf{K}_{i}^{\mathrm{T}} \mathbf{S}_{e}^{-1} \\
& \times\left[\mathbf{y}-P\left(\mathbf{x}_{i}, \mathbf{b}\right)+\mathbf{K}_{i}\left(\mathbf{x}_{i}-\mathbf{x}_{a}\right)\right],
\end{aligned}
$$

where $\mathbf{x}_{a}$ is the a priori assumption for $\mathbf{x}, \mathbf{S}_{a}$ is the a priori uncertainty expressed as the covariance matrix of $\mathbf{x}_{a}, \mathbf{S}_{e}$ is the covariance matrix of the combined measurement uncertainty and forward model error, $\mathbf{b}$ contains additional, fixed model parameters, and $\mathbf{K}_{i}$ is the Jacobian matrix of $P$ linearized around $\mathbf{x}_{i}$. Following the method of Turner and Löhnert (2014), an additional decreasing factor $\gamma_{i}=1000,300,100,30,10,3,1,1, \ldots$ is used: in the case of a bad first guess, those elements of $\mathbf{x}$ are adjusted first that can be obtained best from the observation. This enhances the stability of the retrieval. Uncertainties in $\mathbf{b}$ are expressed by the covariance matrix $\mathbf{S}_{b}$ and can be considered by replacing $\mathbf{S}_{e}$ with $\mathbf{S}_{e}+\mathbf{K}_{b}^{\mathrm{T}} \mathbf{S}_{b} \mathbf{K}_{b}$.

From the Bayesian concept, the uncertainty in the optimal solution can be estimated from

$$
\mathbf{S}_{i}=\mathbf{B}_{i}^{-1}\left(\gamma_{i}^{2} \mathbf{S}_{a}^{-1}+\mathbf{K}_{i}^{\mathrm{T}} \mathbf{S}_{e}^{-1} \mathbf{K}_{i}\right) \mathbf{B}_{i}^{-1},
$$

where

$$
\mathbf{B}_{i}=\left(\gamma_{i} \mathbf{S}_{a}^{-1}+\mathbf{K}_{i}^{\mathrm{T}} \mathbf{S}_{e}^{-1} \mathbf{K}_{i}\right)
$$

The a priori state $\mathbf{x}_{a}$ is used as the starting value $\mathbf{x}_{1}$, and the iteration is stopped when $\gamma_{i}=1$ and the convergence criterion

$$
\left(\mathbf{x}_{i}-\mathbf{x}_{i+1}\right)^{\mathrm{T}} \mathbf{S}_{i}^{-1}\left(\mathbf{x}_{i}-\mathbf{x}_{i+1}\right) \ll N
$$

is met. Then, the individual degrees of freedom for signal $d_{f}$, which describe the number of independent pieces of information that can be obtained from the measurements, can be estimated for each element of $\mathbf{x}$ from the diagonal of the averaging kernel

$$
\mathbf{A}_{i}=\mathbf{B}_{i}^{-1} \mathbf{K}_{i}^{\mathrm{T}} \mathbf{S}_{e}^{-1} \mathbf{K}_{i}
$$

after convergence. The diagonal of $\mathbf{A}_{i}$ consists of the $d_{f}$ values for the elements of $\mathbf{x}$. The total degrees of freedom for signal $D_{f}$ can be obtained from the trace of $\mathbf{A}_{i}$, that is, the sum of $d_{f}$ values down the diagonal of $\mathbf{A}_{i}$. The $D_{f}$ is usually less than the number of observations $M$, because the elements of $\mathbf{y}$ share redundant information.

The optimal-estimation code developed for this study is written in Python. It has been released as open source and is available online (https://github.com/maahn/ pyOptimalEstimation).

\section{e. State vector $\mathbf{x}$}

To simulate the radar Doppler spectrum, the PAMTRA forward operator requires information about particle mass $m(D)$, particle cross-sectional area $A(D)$, and particle number $N(D)$ as a function of particle maximum dimension $D$. In addition, kinematic broadening $\sigma_{k}$ and vertical air motion $w$ are required to account for kinematic effects.

The state vector consists of the quantities introduced in section 2a (see also Table 1 for an overview). To estimate the prior information, $\mathbf{S}_{a}$ and $\mathbf{x}_{a}$ are computed from the covariance and mean, respectively, of the quantities in the ISDAC dataset introduced by Maahn et al. (2015), which contains 1360 ISDAC aircraft observations that were closer than $10 \mathrm{~km}$ to Barrow. The a priori information is obtained from one homogeneous data source that allows for consideration of off-diagonal entries of $\mathbf{S}_{a}$ (covariances) and leads to a consistent definition of particle dimension throughout the dataset. Variance and prior knowledge of $\sigma_{k}$ are combined from the horizontal wind $u$ and $\varepsilon$. The mean and variability of $u$ can be estimated from the ISDAC dataset, but that is not possible for $\varepsilon$, because the TAMDAR instrument only provides an upper boundary for $\varepsilon$ of $10^{-3} \mathrm{~m}^{2} \mathrm{~s}^{-3}$. Maahn et al. (2015) estimated $\varepsilon=10^{-6} \mathrm{~m}^{2} \mathrm{~s}^{-3}$ for ISDAC by comparing forward-modeled radar spectra that are based on in situ data with radar observations. In comparison with the values reported by Shupe et al. (2012) for the Mixed-Phase Arctic Cloud Experiment (MPACE), which also took place in Barrow, this value is small. Therefore, an increased value of $1.25 \times 10^{-4} \mathrm{~m}^{2} \mathrm{~s}^{-3}$ is used as the mean of $\varepsilon$ for the a priori in this study. 
TABLE 1. A priori values and uncertainties of the state vector $\mathbf{x}$ and of derived quantities. Here, PSD is particle size distribution.

\begin{tabular}{|c|c|c|c|c|c|c|}
\hline & Name & Source & Unit & Used scale & Linear prior & $\log _{10}$ prior \\
\hline$D_{m}$ & Mass-weighted diameter of PSD & ISDAC cloud probes & $\mathrm{m}$ & $\log _{10}$ & $0.0010 \pm 0.0006$ & $-3.0 \pm 0.3$ \\
\hline$N_{0}^{*}$ & Intercept parameter of PSD & ISDAC cloud probes & $\mathrm{m}^{-4}$ & $\log _{10}$ & $(1.3 \pm 2.0) \times 10^{7}$ & $7.1 \pm 0.7$ \\
\hline$\mu^{*}$ & Shape parameter of PSD & ISDAC cloud probes & - & $\log _{10}$ & $5.5 \pm 5.1$ & $0.74 \pm 0.40$ \\
\hline$\alpha$ & $\begin{array}{l}\text { Dimensionless intercept factor } \\
\text { of } m(D)\end{array}$ & $\begin{array}{l}\text { Closure from ISDAC } \\
\text { and MMCR data }\end{array}$ & - & Linear & $0.97 \pm 0.20$ & - \\
\hline$b$ & Exponent of $m(D)$ & $\begin{array}{l}\text { Closure from ISDAC } \\
\text { and MMCR data }\end{array}$ & - & Linear & $2.0 \pm 0.2$ & - \\
\hline$c$ & Intercept factor of $A(D)$ & ISDAC cloud probes & $\mathrm{m}^{2-d}$ & $\log _{10}$ & $0.17 \pm 0.35$ & $-0.77 \pm 0.89$ \\
\hline$d$ & Exponent of $A(D)$ & ISDAC cloud probes & - & Linear & $1.9 \pm 0.3$ & - \\
\hline$\sigma_{k}$ & Kinematic broadening & $\begin{array}{l}\text { ISDAC horizontal wind } \\
\text { and TAMDAR }\end{array}$ & $\mathrm{m} \mathrm{s}^{-1}$ & $\log _{10}$ & $0.074 \pm 0.052$ & $-1.1 \pm 0.3$ \\
\hline$w$ & Vertical wind & ISDAC vertical wind & $\mathrm{m} \mathrm{s}^{-1}$ & Linear & $0.04 \pm 0.32$ & - \\
\hline IWC & Ice water content & Derived from $\mathbf{x}$ & $\mathrm{kg} \mathrm{m}^{-3}$ & $\log _{10}$ & $(2.0 \pm 5.0) \times 10^{-5}$ & $-4.7 \pm 1.1$ \\
\hline$N_{\text {tot }}$ & Number of particles & Derived from $\mathbf{x}$ & $\mathrm{m}^{-3}$ & $\log _{10}$ & $(1.8 \pm 3.4) \times 10^{3}$ & $3.3 \pm 0.8$ \\
\hline$r_{\mathrm{eff}}$ & Effective radius & Derived from $\mathbf{x}$ & $\mathrm{m}$ & Linear & $(5.2 \pm 3.2) \times 10^{-4}$ & - \\
\hline
\end{tabular}

For the response functions (section 3 ), the lower $\left(10^{-6} \mathrm{~m}^{2} \mathrm{~s}^{-3}\right)$ and upper values $\left(10^{-3} \mathrm{~m}^{2} \mathrm{~s}^{-3}\right)$ of $\varepsilon$ are investigated as well. The a priori uncertainty of $\log _{10} \varepsilon$ is assumed to be 1.0 as observed by Shupe et al. (2012) for MPACE. Covariances of kinematic variables with microphysical parameters are not considered.

Optimal estimation requires that the uncertainties of $\mathbf{x}_{a}$ (and $\mathbf{y}$ ) be described by a Gaussian distribution with variance as provided in $\mathbf{S}_{a}\left(\mathbf{S}_{e}\right)$. For most elements of $\mathbf{x}$, it is found that the distribution of the logarithmic value follows a Gaussian distribution better. As a consequence, for $D_{m}, N_{0}^{*}, \mu^{*}, c$, and $\sigma_{k}$, the logarithm of the quantities is used instead. Because of the different units of the quantities of $\mathbf{x}$, the correlation matrix belonging to $\mathbf{S}_{a}$ is presented in Fig. 1a instead. Note that the logarithm of the prefactor $c$ of $A(D)$ is highly correlated (correlation coefficient squared $R^{2}=0.99893$ ) with the exponent $d$ for the ISDAC dataset. Even though a high correlation (0.836) between the coefficients of $A(D)$ was also found by Wood et al. (2014), the reason for this almost perfect correlation in the ISDAC dataset remains unclear. It could be related to issues with the measurements, the postprocessing, or the fitting technique. Also the correlation between $N_{0}^{*}$ and $D_{f}$ is high $(-0.804)$.

In addition to these quantities, PAMTRA output depends also but weakly on pressure $p$ and temperature $T$,
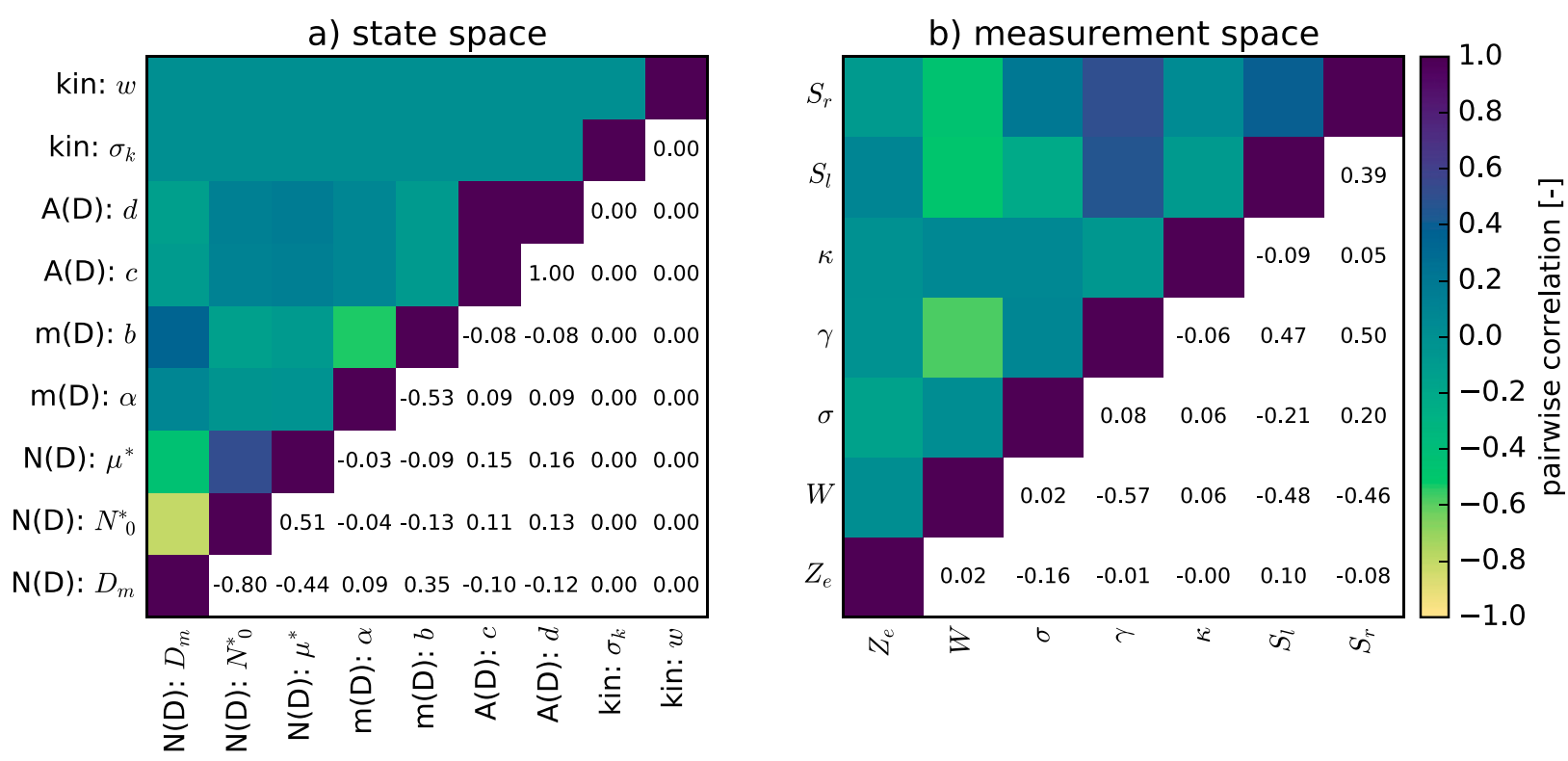

FIG. 1. (a) Correlation matrix of state vector $\mathbf{x}$ used for all profiles, and (b) an example correlation matrix of the measurement vector $\mathbf{y}$ for $\sigma=0.08 \mathrm{~m} \mathrm{~s}^{-1}$. 
which are required for estimation of the particle fall velocity and the scattering properties. In this study, they are obtained from the ISDAC dataset; in a real-world application they can be estimated from radiosondes or model data. Therefore, they are treated as known parameters in the retrieval.

\section{f. Measurement vector $\mathbf{y}$}

In what follows, different configurations of the measurement vectors are evaluated. In all cases, the measurement vector is composed of one or several quantities taken from the moments and slopes of the Doppler spectrum: equivalent radar reflectivity factor $Z_{e}$, mean Doppler velocity $W$, Doppler spectrum width $\sigma$, skewness $\gamma$, and kurtosis $\kappa$ as well as the left slope $S_{l}$ and right slope $S_{r}$.

\section{g. Measurement uncertainty}

The measurement uncertainty and forward-model error are combined in the covariance matrix $\mathbf{S}_{e}$. For the former, values of $0.5 \mathrm{~dB}$ for $Z_{e}$ and $0.1 \mathrm{~m} \mathrm{~s}^{-1}$ for $W$ as well as $\sigma$ have been reported by Widener and Johnson (2005) for the MMCR. Uncertainties for higher moments or the slopes have not been published for the MMCR yet. Therefore, we use a different approach here to estimate the measurement uncertainty for all moments and slopes consistently: We assume that the measurement uncertainty of the MMCR is dominated by random effects (in contrast to a possible bias-see section $5 b$ for a discussion of systematic offsets resulting from wrong calibration). This means that we exclude other possible uncertainty sources such as receiver and transmitter instability. We are confident that we can neglect the former because the variability of the radar noise was found to be very low $(<0.0015 \mathrm{~dB})$, and the latter is monitored for the MMCR in order to adjust the calibration accordingly (Widener and Johnson 2005). Random effects are included in PAMTRA by perturbing each bin of the Doppler spectrum with a random number drawn from an exponential distribution as in Zrnić (1975). Therefore, we can run PAMTRA a sufficient number of times for the same profile and compute the covariance from the spread of the results. With this approach, we obtain the uncertainties not only for the lower moments but also for the higher moments and the slopes. Furthermore, this method allows for estimation of the covariances between the elements of $\mathbf{y}$. These covariances provide a more realistic uncertainty estimate than does a purely diagonal $\mathbf{S}_{e}$ matrix, giving more weight to the measurements (Ebell et al. 2010).

Measurement uncertainties are usually assumed to be constant, but they depend strongly on the signal-to-noise ratio (SNR) and on the number of bins of the Doppler spectrum that are covered by the peak $\left(N_{B}\right)$. In general, observations with highest SNR and largest $N_{B}$ have the smallest $\mathbf{S}_{e}$. To estimate the specific $\mathbf{S}_{e}$ for a particular state $\mathbf{x}$, PAMTRA is run 1000 times for the same $\mathbf{x}$ (we found that running the forward model 1000 times leads to stable results). When using real observations instead of synthetic ones, $\mathbf{x}$ is not known in advance and $\mathbf{S}_{e}$ has to be estimated differently: the Doppler spectrum width $\sigma$ depends on both SNR and $N_{B}$; therefore, we take $\log _{10} \sigma$ (accounting for nonlinear effects by the use of $\log _{10}$ ) as a parameter to choose the appropriate $\mathbf{S}_{e}$ from a lookup table. This lookup table is created by estimating $\mathbf{S}_{e}$ and $\sigma$ for all profiles of the ISDAC dataset. The resulting $\mathbf{S}_{e}$ are sorted into classes depending on $\log _{10} \sigma$, and for every class the mean $\mathbf{S}_{e}$ is calculated. The impact of using a lookup table for $\mathbf{S}_{e}$ instead of estimating $\mathbf{S}_{e}$ from $\mathbf{x}$ on retrieval performance is negligible. Therefore, only the lookup-table method is used in the following.

The measurement uncertainties corresponding to the square root of the diagonal of $\mathbf{S}_{e}$ are presented in Fig. 2 as a function of $\log _{10} \sigma$. It can be seen that the uncertainty for $Z_{e}$ varies between 0.2 and $0.7 \mathrm{~dB}$, which means that we assume lower and higher uncertainties for $Z_{e}$ than were reported by Widener and Johnson (2005) depending on $\sigma$. For $W$ and for $\sigma$ itself, we find, however, significantly lower uncertainty values than $0.1 \mathrm{~m} \mathrm{~s}^{-1}$. Given that the MMCR had a spectral resolution of $0.04 \mathrm{~m} \mathrm{~s}^{-1}$ during ISDAC, an uncertainty of $0.1 \mathrm{~m} \mathrm{~s}^{-1}$ appears to be a very conservative estimate for $W$ (this might be different for observations with a much lower SNR than those investigated in this study-e.g., liquid clouds). This is also confirmed by Matrosov (2011), who found a standard deviation between $W$ observations of ice clouds of an MMCR and a W-band radar of $0.03-0.06 \mathrm{~m} \mathrm{~s}^{-1}$. Given that this corresponds to the difference between two radar systems that were neither completely beam matched nor free of pointing errors, we are confident that the measurement uncertainty of $W$ is considerably lower. Therefore, we stick to our uncertainty estimate for $W$ of $0.01-0.02 \mathrm{~m} \mathrm{~s}^{-1}$ and assume that-at least for the investigated ice clouds-the reported uncertainty value for $\sigma$ is too high as well. For $\gamma, \kappa$, and the slopes, we found very high measurement uncertainties for $\sigma<0.1 \mathrm{~m} \mathrm{~s}^{-1}$ and significantly reduced uncertainties for larger values. We attribute this to a more stable estimation of these quantities for wider peaks.

\section{h. Forward-model error}

In addition to the measurement uncertainty, $\mathbf{S}_{e}$ contains also the forward-model error, which can 
- combined

- - meas. uncertainty only
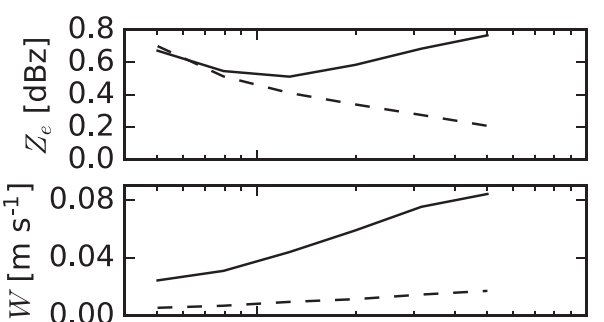

0.04

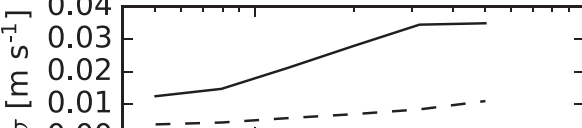

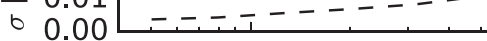
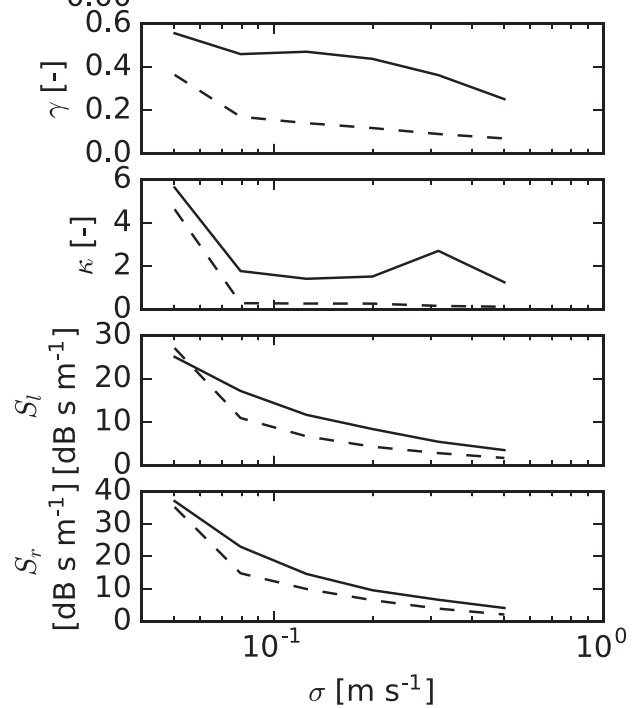

FIG. 2. Measurement uncertainty (dashed) and combined measurement uncertainty and forward-model error (solid) for the quantities of $\mathbf{y}$ as a function of $\sigma$.

propagate to the retrieval solution. Maahn et al. (2015) used $m(D)$ [Eq. (1)] as a closure between aircraft in situ observations and ground-based radar observations for ISDAC. By this, they ensured that potential biases due to scattering assumptions, $v(D)$ parameterization, radar calibration, and aircraft in situ measurements are balanced by $m(D)$. The similarity of the found $m(D)$ coefficients to literature values gives confidence that these biases are small. Because the same $m(D)$ relations are used in this study for the a priori together with the same forward operator, we are confident that our forward operator is generally not biased. Note that Maahn et al. (2015) used the T-matrix approach instead of the SSRG approximation; the equality of both methods for the presented dataset is shown in the online supplemental material.
In addition to biases, there are random errors such as the following:

1) There is discretization error of measured $N(D)$ and $A(D)$. The impact of discretization of $N(D)$ and $A(D)$ was investigated in Maahn et al. (2015). For $N(D)$, no significant impact was found when describing $N(D)$ by a normalized gamma distribution [Eq. (2)], but for $A(D)$ it was found that the approximation of $A(D)$ as a power law leads to a differences in $A$ of up to a factor of 2, which in turn cause biases in $\gamma$ and $\kappa$. These differences are accounted for when running PAMTRA 1000 times. In this way, $\mathbf{S}_{e}$ also contains the forwardmodel error that originates from the description of $A(D)$ using a power law.

2) There is also error in the fall velocity relation $v(D)$. In our forward model PAMTRA, the uncertainty in $A(D)$ described above relates directly to an uncertainty in $v(D)$ of $11 \%$. This is because $A(D)$ is used in PAMTRA solely for estimation of $v(D)$. Therefore, we decided not to consider an additional uncertainty for $v(D)$ although Heymsfield and Westbrook (2010) reported an uncertainty of $25 \%$ for $v(D)$. We expect, however, in practice an error for $v(D)$ of smaller than $25 \%$ because of the large ensemble of simultaneously observed particles.

3) Third, there are errors in the estimation of backscattering cross section $\sigma_{\mathrm{bsc}}$. In this study, a fixed value of 0.6 is used for the aspect ratio AR. Because the two other quantities, which according to SSRG theory determine the ensemble scattering properties, $N(D)$ and $m(D)$ are not fixed but are included in $\mathbf{x}$, we can assume that the forward-model error for estimating $\sigma_{\text {bsc }}$ is determined by the assumption of a fixed AR. Maahn et al. (2015) found that AR is greater than 0.5 on the basis of ISDAC in situ data, and literature values for AR vary between 0.6 (Hogan et al. 2012) and 0.7 (Tyynelä et al. 2011). Therefore, we draw AR from a normal distribution with mean 0.6 and standard deviation 0.1 when running PAMTRA 1000 times to estimate $\mathbf{S}_{e}$. Because AR only affects the estimation of $\sigma_{\mathrm{bsc}}$ within PAMTRA, this approach is equivalent to assuming an uncertainty for the estimation of $\sigma_{\mathrm{bsc}}$ directly. Note that this method includes the implicit assumption that the bulk scattering properties of ice particles depend only on $D, m$, and AR. This will likely lead to biases when applying the retrieval to clouds consisting of particles other than these for which SSRG was developed, for example, pristine or heavily rimed particles. For the ISDAC dataset, we are, however, confident that heavily rimed particles were rare because observations 
containing SCLW were filtered. Also pristine particles were-with the exception of at cloud top-likely scarce given that cloud depths were typically larger than $1000 \mathrm{~m}$.

Using the same data and forward-operator set in Maahn et al. (2015) and in this study allows for a consistent handling of the assumptions and uncertainties associated with the ISDAC dataset. The forward-model error combined with the measurement uncertainty is presented in Fig. 2. Inclusion of the forward-model error leads to clearly enhanced uncertainties for all elements of y. Only for small $\sigma$, the forward-model error does not increase the combined error for $Z_{e}, S_{l}$, and $S_{r}$ that is related to the dominating measurement uncertainty for small SNR. The measurement uncertainty is even slightly larger than the combined error for two data points, which we attribute to the averaging of $\mathbf{S}_{e}$. Because $Z_{e}$ does not depend on $v(D)$ and the assumed uncertainty, the increased values for $Z_{e}$ depend only on the assumed uncertainty for AR. For small $\sigma$ values, which typically coincide with small $Z_{e}$ values, the forward-model error does not contribute to the uncertainty estimate of $Z_{e}$, which is most likely related to the domination of smallparticle scattering in the Rayleigh regime. For larger $\sigma$, the forward-model error increases, which is probably related of the presence of larger particles for which the scattering properties depend stronger on AR. In contrast, $W$ depends only on the assumed uncertainties of $v(D)$ [or rather $A(D)$ ]. Because a relative error is assumed, the forward-model uncertainty is increasing with larger $\sigma$ values that are typically related to larger $W$ values. The full $\mathbf{S}_{e}$ matrix corresponding to the combined measurement and forward-model error is shown in Fig. $1 \mathrm{~b}$ for $\sigma=0.08 \mathrm{~m} \mathrm{~s}^{-1}$.

To investigate the possibility that the discussion of forward-model and/or measurement errors is incomplete or that uncertainties have been underestimated, we analyze in section $5 \mathrm{c}$ the retrieval performance under the assumption that $\mathbf{S}_{e}$ is 4 times as large; this corresponds to a duplication of the uncertainties.

\section{Response functions}

The response functions of the forward model that show the impact of the parameters on the moments and slopes of the radar Doppler spectrum are now investigated. Note that this section follows Maahn (2015), with minor modifications.

The response functions are presented in Fig. 3. Because kinematic broadening $\sigma_{k}$ can lead to peaks that are more Gaussian shaped and can potentially reduce the response functions of other elements of $\mathbf{x}$, the response functions are estimated for three different values of $\sigma_{k}$. Because $\sigma_{k}$ depends on the horizontal wind and on $\varepsilon$, this is implemented by assuming a fixed horizontal wind of $10 \mathrm{~m} \mathrm{~s}^{-1}$ and three different values of $\varepsilon\left(1 \times 10^{-6}, 1.25 \times 10^{-4}\right.$, and $\left.1 \times 10^{-3} \mathrm{~m}^{2} \mathrm{~s}^{-3}\right)$ covering the range of $\varepsilon$ values found by Shupe et al. (2008) for the MPACE campaign in Barrow. Each response function is estimated 1000 times to assess its measurement uncertainty, that is, to investigate whether the moments and slopes are stable with respect to radar noise. The resulting uncertainty range indicated by the 10 th and 90th percentiles is shown in addition to the median value.

Reflectivity $Z_{e}$ (Fig. 3a) depends strongly on $D_{m}$ and on $N_{0}^{*}$ but very little on the shape parameter $\mu^{*}$. Furthermore, $Z_{e}$ is affected by the coefficients of the normalized mass-size relation $\alpha$ and $b ; Z_{e}$ is not affected by the area-size relation and the vertical air motion $w$ because these quantities influence only the fall velocity of the particles.

Mean Doppler velocity $W$ (Fig. 3b) depends on both mass-size and area-size coefficients $(\alpha, b, c$, and $d)$ through the fall-velocity parameterization, with the response of $W$ to $c$ and $d$ being particularly strong. For $N(D), W$ depends only on $D_{m}$ and not on $N_{0}^{*}$ because $N_{0}^{*}$ scales $N(D)$ only in the direction of the ordinate. The shape parameter $\mu^{*}$ has only little impact on $W$. As expected, vertical air motion $w$ affects $W$ linearly.

In contrast to $Z_{e}$ and $W$, Doppler spectrum width $\sigma$ (Fig. 3c) depends strongly on the assumed turbulence level, which causes an offset of $\sigma$ but has little influence on the response functions of the various microphysical parameters. Apart from the coefficients of the area-size and mass-size relations, with a particularly strong response to $b, \sigma$ also depends on the shape parameter $\mu^{*}$ but does not depend on $D_{m}$ and $N_{0}^{*}$.

Skewness $\gamma$ (Figs. 3d) is influenced by all of the parameters but $N_{0}^{*}$. The response is small in comparison with the lower-order moments (with the exception of $b$ ), and the interval of estimated uncertainty $(\sim 0.25)$ is often of a size that is similar to that of the response. For $b$ only is the response greater than 1 , but it is also very nonlinear and not monotonic. In theory, $\gamma$ should not depend on $w$, but a small, sinuous response can be seen that is related to discretization effects. When configuring the radar simulator with a higher Doppler spectrum resolution (e.g., 512 FFT points), the sinuous response vanishes (not shown). Because increasing turbulence leads to a more Gaussian shape of the peak, which typically has a skewness value that is near zero, the 

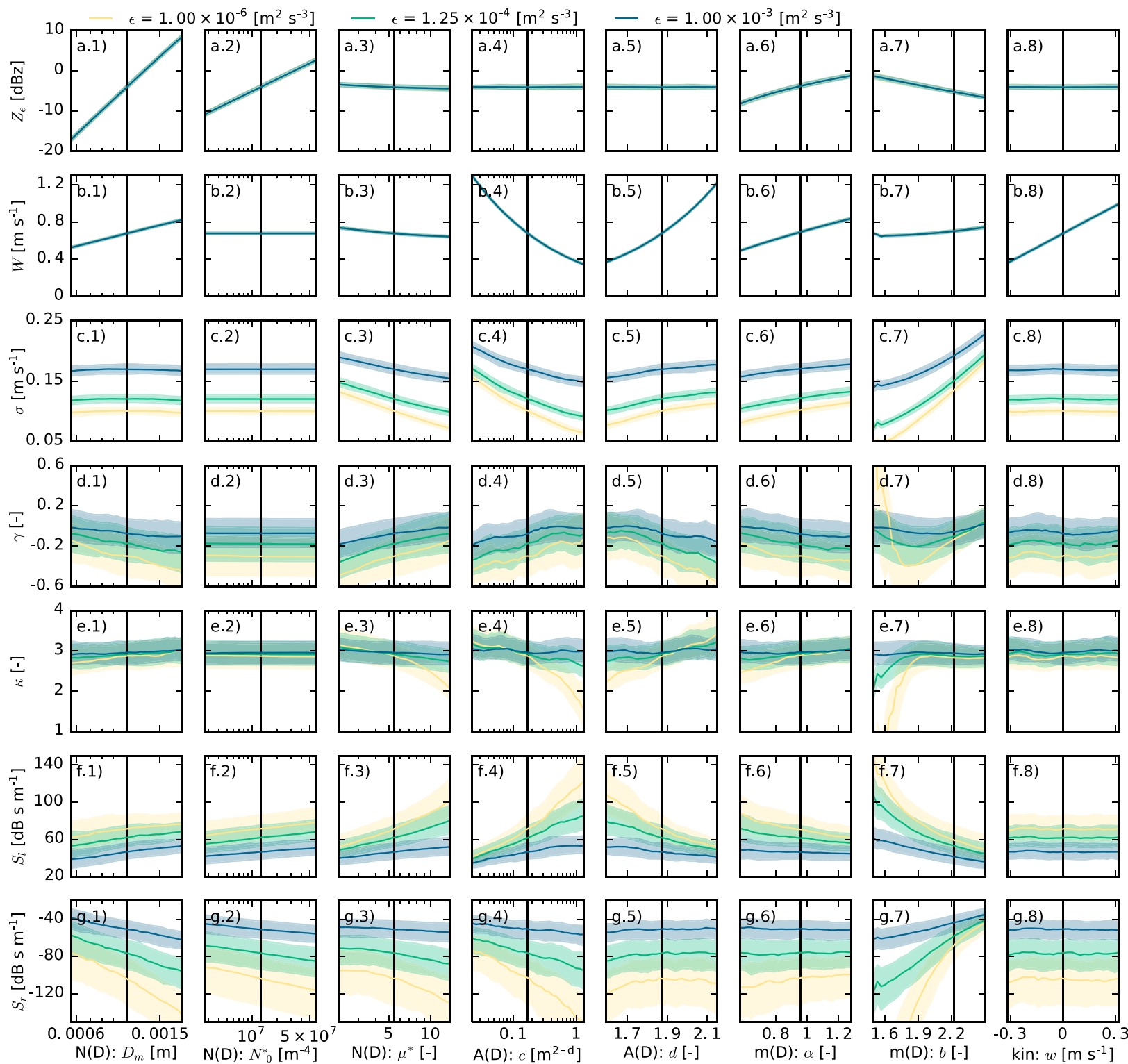

FIG. 3. Impact of the parameters of the state vector (columns) on moments and slopes of the radar Doppler spectrum (rows) for values of the eddy dissipation rate of $10^{-6}$ (yellow), $1.25 \times 10^{-4}$ (green), and $10^{-3}$ (blue) $\mathrm{m}^{2} \mathrm{~s}^{-3}$. The uncertainties of moments and slopes (10th and 90th percentiles) caused by radar noise are indicated as colored areas around the median values' lines. The black vertical line denotes the mean value of the parameters in the ISDAC dataset.

response functions are reduced for the medium turbulence level. For the high turbulence level, the response of $\gamma$ is less than the estimated uncertainty range for all parameters even though also the uncertainty range of $\gamma$ is reduced.

Kurtosis $\kappa$ (Fig. 3e) is mainly determined by the shape parameter $\mu^{*}$ and the coefficients of the area-size and mass-size relations, leading to a response of up to 3 . This is larger than the uncertainty of $\kappa(\sim 0.4)$. The response to $b$ is, however, saturated for values of greater than 1.9. Similar to $\gamma$, the response of $\kappa$ is reduced for the medium turbulence level. For the highest turbulence level, $\kappa$-like a Gaussian distribution-generally has a value of 3; the response functions are greatly reduced and are smaller than the uncertainties.

The left slope $S_{l}$ (Fig. 3f) responds to all parameters except $w$, but only for $\mu^{*}, b, c$, and $d$ is the response clearly greater than the uncertainty of up to $20 \mathrm{~dB} \mathrm{~s} \mathrm{~m}^{-1}$. In comparison with $\gamma$ and $\kappa$, the uncertainty appears larger because the slopes are obtained from the Doppler spectrum in logarithmic scale, which increases the impact of radar noise. The moments, on the contrary, are 
estimated from the spectrum in linear scale. Similar to $\gamma$ and $\kappa$, the response functions are reduced for the medium and high turbulence intervals and are often within or close to the noise estimate. The uncertainty estimate is, however, reduced for higher turbulence, which is probably related to the smoothing effect of turbulence. Hence, $S_{l}$ might be even more exploitable by the retrieval in higher turbulence conditions despite the smaller response functions.

The response functions of the right slope $S_{r}$ (Fig. 3g), which is mainly determined by the fastest-falling particles, are similar to $S_{l}$ with respect to noise. For $D_{m}, b$, and $c$, the responses are largest and are significantly larger than the uncertainty.

\section{Retrieval results}

To investigate the benefit of adding higher-order moments and slopes to a radar retrieval, an optimalestimation-based retrieval is applied to synthetic radar observations based on ISDAC in situ profiles using the parameterizations introduced in section 2a. The training dataset for a priori estimation is based on Maahn et al. (2015), who used only aircraft observations that were closer than $10 \mathrm{~km}$ to Barrow (Table 1). For separation of the training and the validation datasets, the retrieval is applied to 1371 synthetic radar observations obtained at distances from 40 to $10 \mathrm{~km}$ from Barrow. Using a maximum distance of $40 \mathrm{~km}$ results in almost equal sizes for the training and validation datasets.

The percentage of the 1371 ISDAC profiles that converge successfully to a solution $P$ is presented in Fig. 4. The values of $P$ vary between $80 \%$ and $100 \%$. A profile does not converge if either the convergence criterion [Eq. (16)] is not met within 30 iteration steps or the retrieval iterates to a state that either is below radar sensitivity or is physically inconsistent (e.g., ice crystals with a density that is greater than that of pure ice) and that cannot be forward modeled. Addition of higherorder moments or slopes and omission of lower-order moments reduces $P$, which is most likely related to the greater noise in higher-order moments and slopes as well as their less linear response to parameters describing microphysical properties.

\section{a. Total degrees of freedom for signal}

The total degrees of freedom for signal $D_{f}$ that can be obtained from radar observations are presented in Fig. 4. As expected, a retrieval exploiting only reflectivity can achieve only $D_{f}=1.0$. Addition of $W$ (using only $Z_{e}$ and $W$ is called LM in the following) adds 1.0 to $D_{f}$. If $\sigma$ is also included, $D_{f}$ reaches values around 2.8 depending on the profile. When adding higher-order

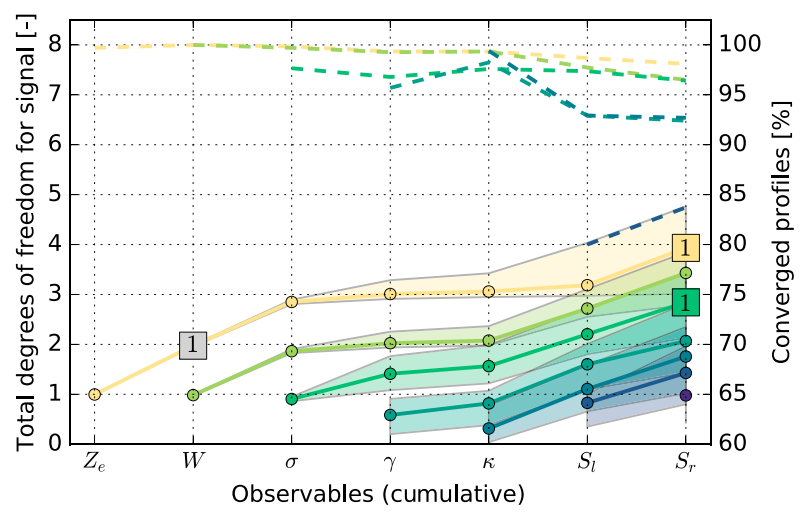

FIG. 4. Mean (solid) and 10th and 90th percentile (shaded area) of total degrees of freedom for signal $D_{f}$ obtained for the 1371 synthetic observations. The different lines are for different, cumulative sets of measurement variables; e.g., the yellow line shows $D_{f}$ of a retrieval only with $Z_{e}$ (first point), for a retrieval with $Z_{e}$ and $W$ (point marked with a 1 in a gray box), and so forth. The first point of the light-green line is for a configuration only with $W$, the second point is for $W$ and $\sigma$, and so forth. For the configurations marked with a 1 in a box, the retrieval solutions are investigated in detail. The dashed lines denote the percentage of retrievals of the different configurations that converged successfully.

moments $\gamma$ and $\kappa$ to the retrieval, the median of $D_{f}$ increases only by $0.1-0.2$ per moment. For some profiles, however, $\gamma$ and $\kappa$ can contribute a multiple as can be seen from the spread. This is probably due to the reduced response functions of the higher-order moments for stronger turbulence: higher-order moments contain exploitable information only, if the radar peak is not Gaussian shaped. This is similar for the slopes. For some profiles, $S_{l}$ and $S_{r}$ do not contribute any information to the retrieval, but on average they add 0.1 and 0.7 , respectively, to $D_{f}$. The greater additional information content of $S_{r}$ in comparison with $S_{l}$ is probably related to the sensitivity of $S_{r}$ to large, fast particles. Since fall velocity depends on particle mass and cross-sectional area, this reveals microphysical properties of these particles that cannot be captured by other moments. The $S_{l}$, in contrast, describes the slowly falling particles whose fall velocities converge to zero with decreasing size. Therefore, the fall velocity does not reveal microphysical properties for these particles. When using all moments and slopes (AM) together, $D_{f}$ is $3.9 \pm 0.9$.

When $Z_{e}$ is removed from the retrieval, $D_{f}$ is reduced by approximately 1 as long as $S_{l}$ is not added to $\mathbf{y}$. Then, $D_{f}$ is only reduced by 0.5 . Therefore, it can be concluded that $S_{l}$ can partly replace $Z_{e}$ because they share partly redundant information. This is different than for $S_{r}$, which is particularly sensitive to large particles with large fall velocities, and therefore addition of $S_{r}$ always increases $D_{f}$ by $0.5-0.7$. 
A retrieval consisting only of $\sigma$ to $S_{l}$ (HM) can achieve a value of $D_{f}$ around $2.8 \pm 0.7$, which is approximately 1.1 less than when using AM. Configurations without $Z_{e}$ and $W$ have in general a high spread of up to 0.7 around the mean value for $D_{f}$.

Using a retrieval that is based only on a single higherorder moment or slope shows that $D_{f}$ values of $0.3-1.0$ are reached. This means that the reason for the small increase in $D_{f}$ when adding $\gamma$ or $\kappa$ to a lower-ordermoments retrieval is not that $\gamma$ and $\kappa$ do not contain any information but rather that this information is partly redundant with that included in the lower-order moments, particularly in $W$ and $\sigma$.

\section{b. Using additional frequencies}

Here, the benefit of adding additional frequencies to the measurement vector is investigated. For this, the impact of a dual-frequency ( $\mathrm{Ka}$ and $\mathrm{W}$ bands) combination as well as a triple-frequency $(\mathrm{Ku}, \mathrm{Ka}$, and $\mathrm{W}$ band) configuration is studied. For simplicity, beamwidth, Nyquist range, number of FFT points, and radar noise characteristics of all frequencies were configured with respect to the MMCR specifications. This might underestimate the obtained $D_{f}$ because kinematic broadening $\sigma_{k}$ can be directly retrieved from $\sigma$ if radars with different beam widths are used (Maahn and Kollias 2012). When applied to real W-band observations, attenuation has to be accounted for even though attenuation for ice and snow is low in the $\mathrm{W}$ band (Nemarich et al. 1988).

The impact on $D_{f}$ of using two or three frequencies is presented in Fig. 5 for three configurations: LM (lower moments $Z_{e}$ and $\left.W\right), \mathrm{HM}\left(\sigma, \gamma, \kappa, S_{l}\right.$, and $\left.S_{r}\right)$, and AM (all moments and slopes). It can be seen that the use of additional frequencies increases $D_{f}$ up to 5.4. Using AM in a single-frequency retrieval leads to a $D_{f}$ value of 3.9, which is $1.2(1.0)$ more than when using a dual-frequency (triple frequency) retrieval with LM with multiple frequencies. Note that this additional information, however, has to be used to retrieve $\sigma_{k}$ as well, which is not required for the LM configuration. Also when using a dual- or triple-frequency configuration, the inclusion of higher-order moments and slopes into the retrieval is beneficial because $D_{f}$ rises faster with the number of frequencies for AM than for LM. If only HM are used, the retrieved $D_{f}$ is between the configurations with AM and LM.

The increase of $D_{f}$ by adding a second frequency is greater than when adding a third one. This is especially true for the LM configuration for which only a little additional information can be obtained for most cases when using a third frequency. This is in contrast to the findings of, for example, Leinonen and Moisseev (2015)

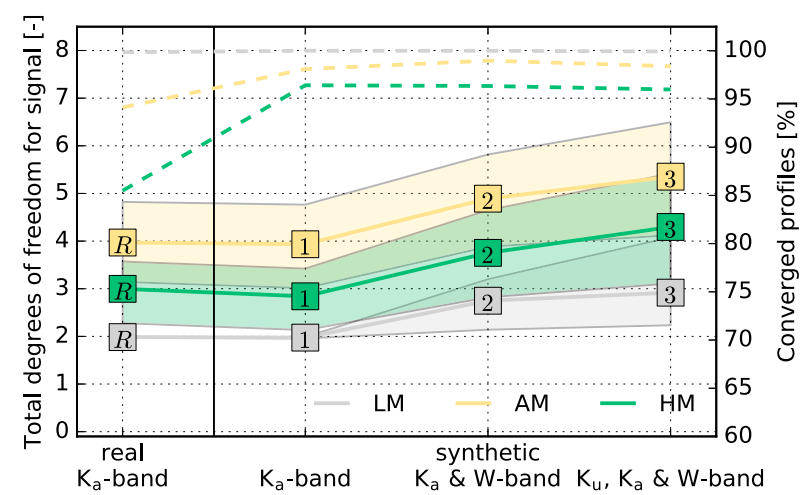

FIG. 5. Mean (solid) and 10th and 90th percentile (shaded area) of total degrees of freedom for signal $D_{f}$ obtained for the three retrieval configurations LM (gray), HM (green), and AM (yellow) as a function of observations used: real single-frequency observations ( $R$ in a box) as well as synthetic single-, dual-, and triplefrequency observations $(1,2$, and 3 , respectively, in the boxes). The dashed lines indicate the percentage of converged profiles.

and Kneifel et al. (2015), who found information in the triple-frequency signatures of reflectivity for aggregation and riming. This is most likely because our dataset contains mostly cloud ice and only a few precipitating snow particles because the aircraft was probing above cloud base most of the time. Also heavily rimed particles are expected to be infrequent in the dataset because data that contain larger amounts of supercooled water are filtered out. It is also unclear whether the SSRG approximation is able to reproduce triple-frequency signatures or whether the coefficients found by Hogan and Westbrook (2014) have to be altered to represent heavily aggregated and/or rimed particles better [see also Kneifel et al. (2016)].

\section{c. Application to real radar data}

So far, retrieval performance was only investigated by using synthetic radar observations. For the Ka band, real radar observations from the MMCR in Barrow are available during ISDAC. To make sure that the real dataset contains ice clouds with microphysical properties comparable to the synthetic dataset, only the radar observations that are closest in time and space to ISDAC in situ observations of the training dataset are analyzed. The MMCR dataset is offset corrected as proposed by Protat et al. (2011) and is filtered for values of liquid water path of greater than $0.1 \mathrm{~kg} \mathrm{~m}^{-2}$, which is the detection threshold of the used microwave radiometer retrieval (Cadeddu 1993). For a cloud with $2.5-\mathrm{km}$ thickness, $0.1 \mathrm{~kg} \mathrm{~m}^{-2}$ of liquid corresponds to approximately $-32 \mathrm{dBz}$. Therefore, the impact of SCLW on the radar moments and slopes can be neglected. The radar dataset contains 1354 profiles, and $D_{f}$ is estimated for 

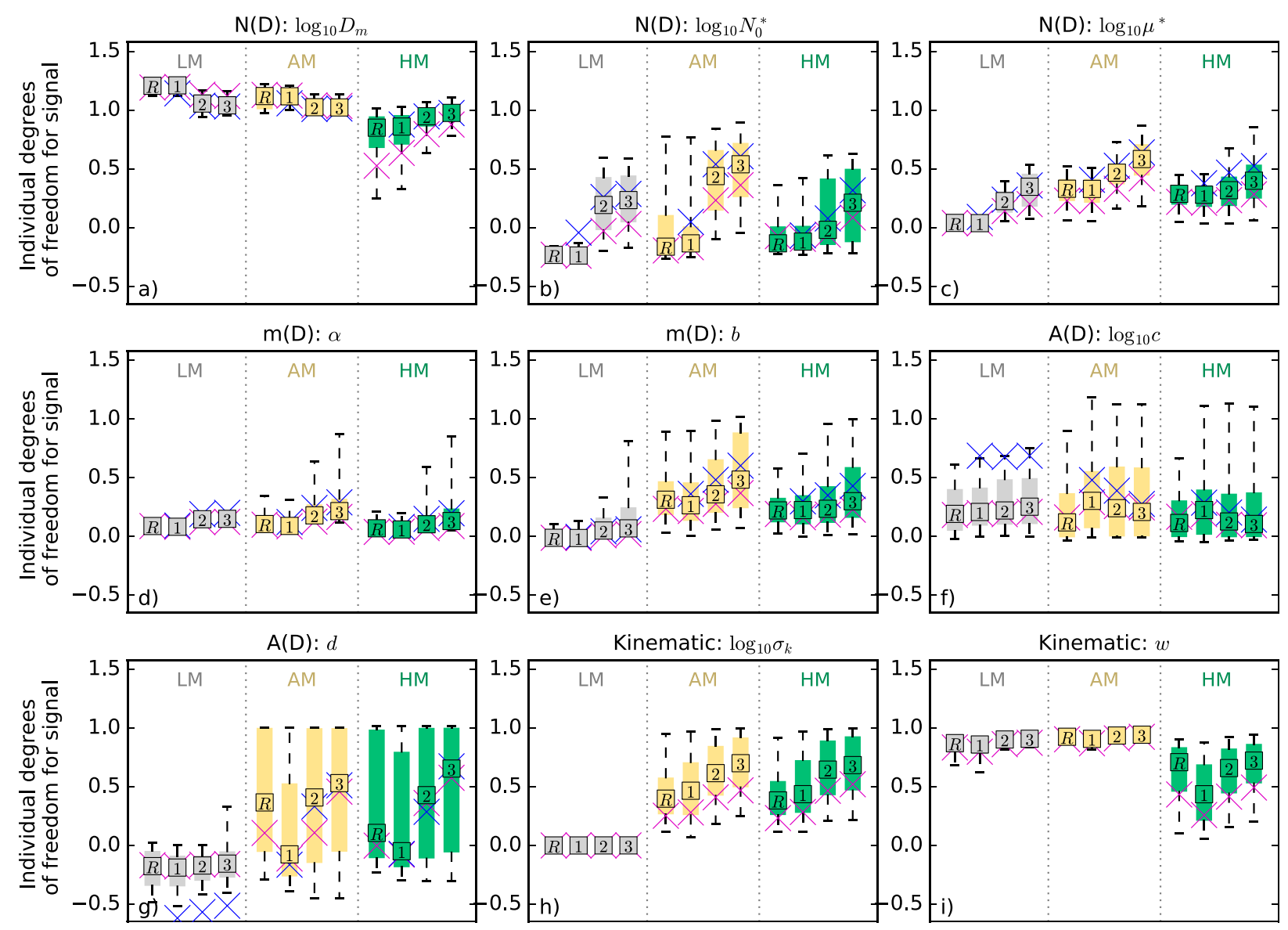

FIG. 6. Box-and-whisker plots showing individual degrees of freedom for signal $d_{f}$ that can be achieved for the elements of $\mathbf{x}$. The boxes indicate the 25th and 75th percentiles, and the whiskers show the 5th and 95th percentiles. Note that the color indicates the set of moments and slopes (gray: LM, yellow: AM, and green: HM) and the symbol indicates the median for real (label $R$ for Ka band) and synthetic radar observations (label 1 for Ka band; label 2 for $\mathrm{Ka}$ and $\mathrm{W}$ band; label 3 for $\mathrm{Ku}, \mathrm{Ka}$, and $\mathrm{W}$ band). The blue $\times$ indicates the impact of previous knowledge of $w$ and $\sigma_{k}$ on the retrieval; the magenta $\times$ is for quadrupling $\mathbf{S}_{e}$ corresponding to a doubling of measurement and forwardmodel errors.

these profiles and the three retrieval configurations of LM, HM, and AM (Fig. 5). It can be seen that $D_{f}$ is as large as for the synthetic observations. This result shows that retrievals using HM or AM are feasible in practice and that the forward model works sufficiently well. Only the percentage of converged profiles $P$ is reduced by $11 \%$ and $4 \%$ for $\mathrm{HM}$ and $\mathrm{AM}$, respectively. This is most likely related to measurement effects that were not considered in the measurement uncertainty such as nonuniform beam filling but can also be related to forward-model errors.

\section{d. Individual degrees of freedom for signal}

The individual degrees of freedom for signal $d_{f}$ for the elements of $\mathbf{x}$ can be estimated from the diagonal elements of $\mathbf{A}$. For the synthetic Ka-band retrieval, best results are obtained for the AM configuration (Fig. 6). Even though the LM configuration features, on average, the lowest values of $d_{f}$, the spread is usually smallest, indicating the stability of the retrieval. Negative $d_{f}$ values and values that are larger than 1 occur for $D_{m}$ and $N_{0}^{*}$ as well as for $c$ and $d$. This is due to the high correlation of these pairs, and these pairs have to be considered together. The sum of both $d_{f}$ of each pair is always smaller than 1.

As expected, no information about $\sigma_{k}$ can be obtained from the LM. Moreover, $d_{f}$ is low for $N_{0}^{*}, \mu^{*}, \alpha$, and $b$, when using only LM. Using AM increases $d_{f}$ for all variables except $D_{m}$ (for which $d_{f}$ is generally very high) and $\alpha$. The generally low $d_{f}$ values for $\alpha$ are in contrast to the clear response functions of $Z_{e}$ and $W$, showing that not all state quantities can be derived unambiguously. HM respond only little to $\alpha$, which implies that there is little benefit when adding higher-order moments and slopes. The increase of $d_{f}$ by using AM is particularly strong for $\mu^{*}, \sigma_{k}, b$, and $d$. 

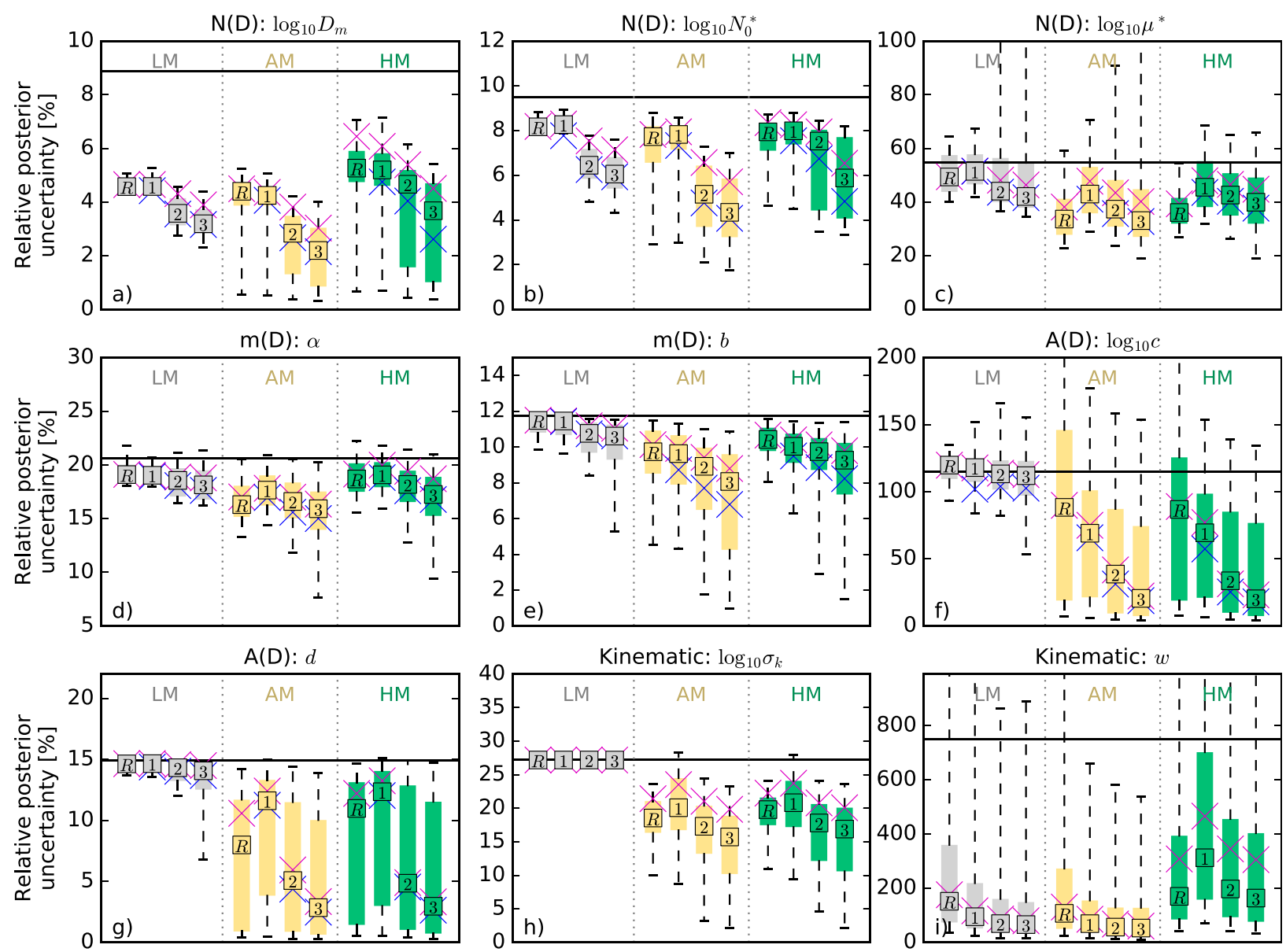

FIG. 7. Relative uncertainty of the solution for the elements of $\mathbf{x}$. Colors and symbols are as in Fig. 6. The thick horizontal line indicates the a priori uncertainty.

The spread of $d_{f}$ is strongly increased when using HM and/or multiple frequencies. This shows the noisiness of $\mathrm{HM}$ and that they do not work for all conditions equally well. Another reason is likely that the retrieval might distribute the information content among the variables differently depending on the profile. For example, for $\kappa$ the response function of $b$ saturates for values larger than 1.9 , which might reduce $d_{f}$ of $b$ for profiles with $b>1.9$.

The impact of using additional frequencies is particularly large for $N_{0}^{*}$ if only LM are used. This is counterintuitive at first sight, because $N_{0}^{*}$ is expected to cancel out from the dual-wavelength ratio (e.g., Kneifel et al. 2011). The reason is probably that the availability of two reflectivity values allows one to retrieve not only $D_{m}$, but also $N_{0}^{*}$ unambiguously. Also for $\mu^{*}, d_{f}$ is increased by approximately $0.2-0.3$ for both LM and AM. For $\alpha, b$, $d$, and $\sigma_{k}$, the increase of $d_{f}$ by adding additional frequencies is much more pronounced when using AM than when using only LM. The decrease of $d_{f}$ for $D_{m}$ is overcompensated by the increase of the highly correlated $N_{0}^{*}$. Except for $D_{m}$ and $w$, there is no variable that does not benefit from adding additional frequencies.

Using only HM gives mostly results between LM and $\mathrm{AM}$, but the spread is largest, indicating the variability of how much information can be obtained from HM among the individual profiles. Applying the retrieval to real observations gives results very similar to the synthetic single-frequency configuration. Only between the highly correlated pair $c$ and $d$ is the information distributed differently among the variables when using $\mathrm{HM}$ or AM.

\section{e. Retrieval uncertainty}

The relative uncertainty of the retrieval solution is shown together with the relative a priori uncertainties in Fig. 7 for the individual elements of $\mathbf{x}$. Note that the uncertainty of the variables treated in logarithmic scale by the retrieval $\left(D_{m}, N_{0}^{*}, \mu^{*}, c\right.$, and $\left.\sigma_{k}\right)$ is expressed logarithmically as well. Because $\log _{10} c$ and $w$ are varying 

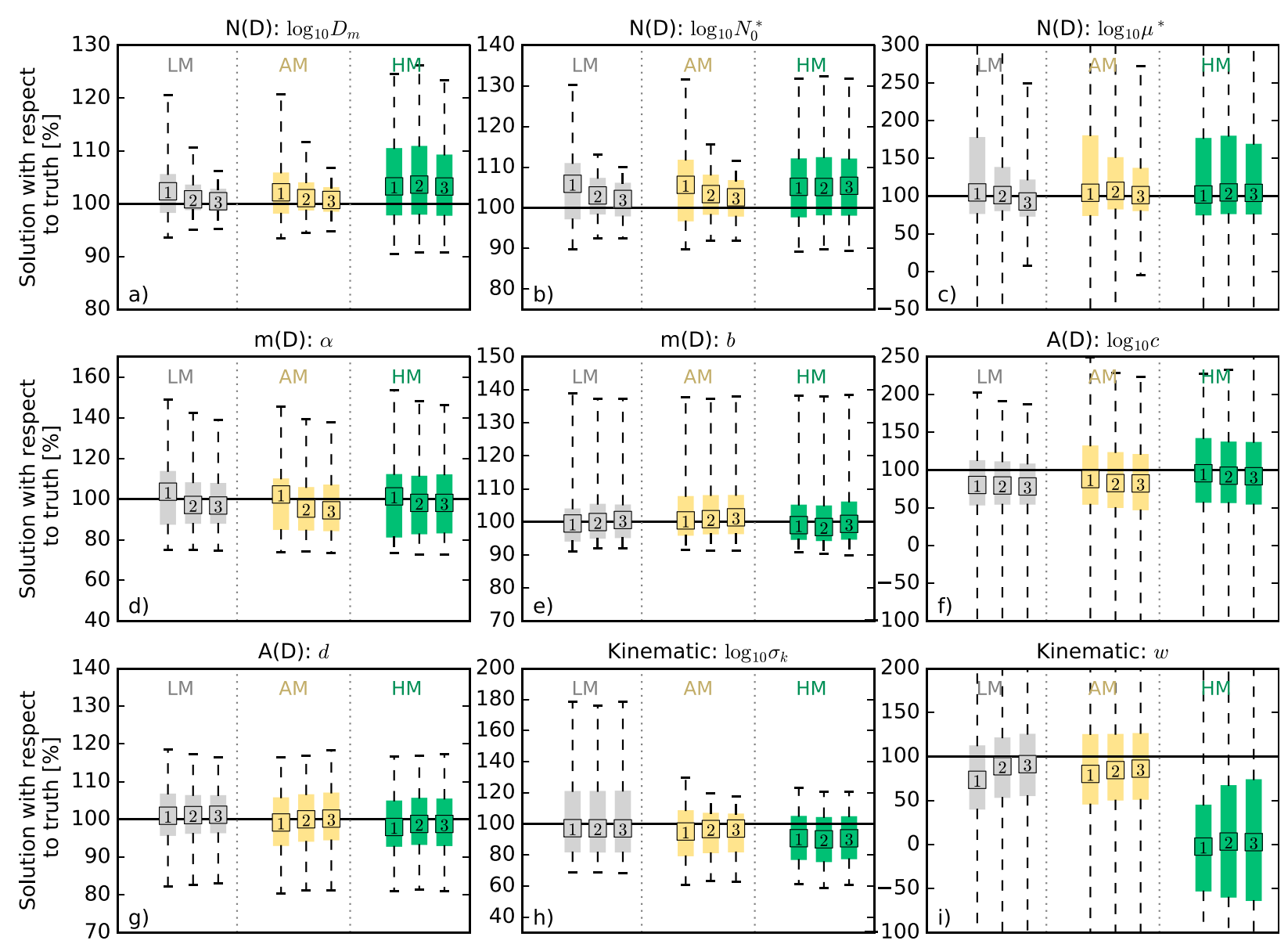

FIG. 8. Solution with respect to truth, in percent. Colors and symbols are as in Fig. 6. The thick horizontal line indicates a bias of zero.

around 0 , their relative uncertainty can reach very high numbers. The absolute, prior uncertainties of $\log _{10} c$ and $w$ are 0.89 and $0.32 \mathrm{~m} \mathrm{~s}^{-1}$, respectively (Table 1 ), and the retrieved absolute uncertainties are accordingly smaller.

Similar to $d_{f}$, the impact of using AM and adding more frequencies to the retrieval can be clearly seen. The uncertainty of some variables depends more on the number of frequencies $\left(D_{m}, N_{0}^{*}\right.$, and $\left.w\right)$, while others can be better obtained when using $\operatorname{AM}\left(\alpha, b, c, d\right.$, and $\left.\sigma_{k}\right)$. For all variables except $\alpha$ and $b$, the median of the uncertainty is more than $50 \%$ smaller than the a priori knowledge when using AM and three frequencies. The small improvement for $\alpha$ can be explained by the fact that not more than 0.2 for $d_{f}$ can be retrieved. For $\alpha$ and $b$, the small a priori uncertainties of 0.20 and 0.24 , respectively, have to be accounted for.

When using multiple frequencies, but in particular when using HM, the spread of uncertainties among retrieval solutions is very large. This highlights, similar to $d_{f}$, that the advantage of using higher-order moments and slopes but also of multiple frequencies depends on the profile. Similar to $d_{f}$, the results for the real observations are on average very similar to the results of the synthetic single-frequency observations.

When using only HM, the median of the relative uncertainty is between LM and AM for $\alpha, b, c, d$, and $\sigma_{k}$. For $D_{m}, N_{0}^{*}$, and $w$, the retrieval works better when using only LM than when using only HM.

\section{f. Retrieval bias}

For the synthetic observations, it is also possible to investigate whether the retrieval solution is in agreement with the aircraft in situ profile that was used to simulate the observations (Fig. 8). In general, the bias between truth and retrieved state is below $5 \%$, with the exception of $c$ and $w$. This result is related to the fact that these quantities typically have values close to zero, which leads to large relative biases even though the absolute biases are small. The spread of parameters describing $N(D)$ is slightly increased for singlefrequency retrievals but reduced for multifrequency retrievals. 

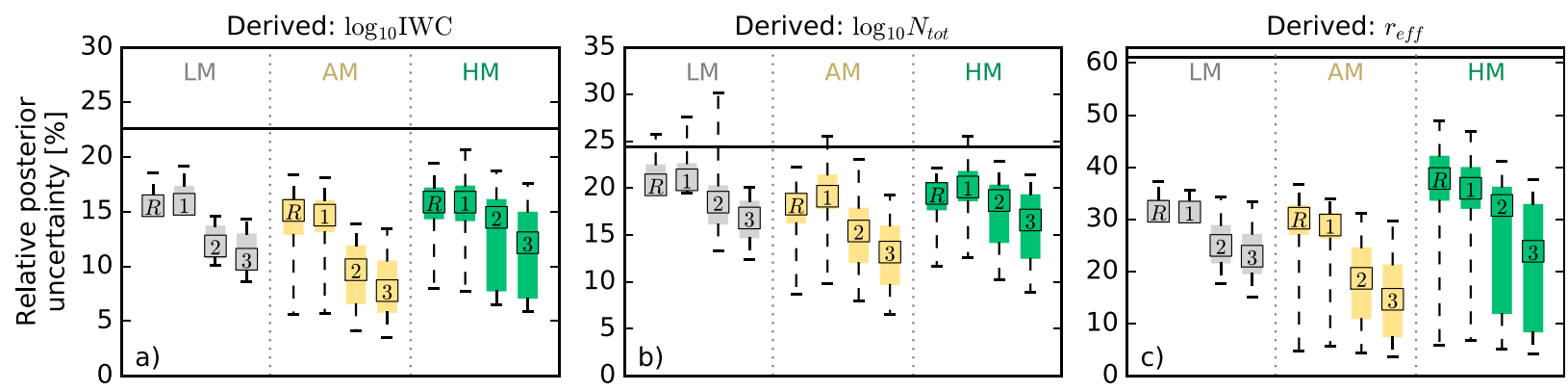

FIG. 9. Relative uncertainty of the solution for the derived variables IWC, $N_{\text {tot }}$, and $r_{\text {eff }}$ Colors and symbols are as in Fig. 6 . The thick horizontal line indicates the a priori uncertainty.

\section{g. Derived values}

The uncertainty of the retrieval solution can also be estimated for variables derived from $\mathbf{x}$ such as

$$
\begin{aligned}
\mathrm{IWC} & =\sum N\left(D, N_{0}^{*}, D_{m}, \mu^{*}\right) m(D, \alpha, b) \Delta D, \\
N_{\mathrm{tot}} & =\sum N\left(D, N_{0}^{*}, D_{m}, \mu^{*}\right) \Delta D, \text { and } \\
r_{\mathrm{eff}} & =\frac{\sum(D / 2)^{3} N\left(D, N_{0}^{*}, D_{m}, \mu^{*}\right) \Delta D}{\sum(D / 2)^{2} N\left(D, N_{0}^{*}, D_{m}, \mu^{*}\right) \Delta D},
\end{aligned}
$$

with Gaussian error propagation (Fig. 9). IWC and $N_{\text {tot }}$ are investigated in logarithmic scale because they can span several magnitudes. The derived variables depend heavily on $D_{m}$ and $N_{0}^{*}$, which benefit less from the use of AM than other quantities. Therefore, the impact on the investigated derived quantities of adding higher-order moments and slopes to a single-frequency retrieval is smaller than when using LM but adding more frequencies. When using AM and multiple frequencies, the uncertainty (see Table 1) of $\log _{10}$ IWC and $r_{\text {eff }}$ can be reduced by a factor of 3 with respect to the prior uncertainty. For $\log _{10} N_{\text {tot }}$, an improvement of $40 \%$ can be achieved.

\section{Retrieval modifications}

\section{a. Prior knowledge of kinematics}

A potential improvement of the retrieval would be the inclusion of continuity in time and space because microphysical and, in particular, kinematic variables do not change drastically between two range gates and/or time steps. In practice, this could be achieved by updating the a priori knowledge depending on neighboring/ previous observations and the correlation length of the corresponding parameters (analogous to a Kalman filter). Because only the potential of this modification is evaluated, here a different approach is chosen: we investigate how the retrieval changes if $w$ and $\sigma_{k}$ are excluded from the retrieval and assumed to be known from another source (two-step retrieval). Uncertainties of externally retrieved $w$ and $\sigma_{k}$ are expressed by $\mathbf{S}_{b}$. For $\log _{10} \sigma_{k}$, Shupe et al. (2012) report an uncertainty of 0.4 , which is assumed to be the 2-sigma uncertainty of $\log _{10} \sigma_{k}$. For $w$, Kalesse and Kollias (2013) developed a retrieval for cirrus clouds with a 2-sigma uncertainty of $0.3 \mathrm{~m} \mathrm{~s}^{-1}$. Covariance elements of $\mathbf{S}_{b}$ are neglected. This corresponds to relative prior uncertainties of $349 \%$ and $18 \%$ for $w$ and $\sigma_{k}$, respectively, which is similar to their posterior uncertainty when including the kinematic variables into the retrieval. Note that this modification can only be applied to the synthetic retrieval configurations.

Exclusion of the kinematic variables from the retrieval reduces the total $D_{f}$ by $0.6-1.3$ depending on configuration when using AM and HM (not shown). This indicates that the advantage of knowing $w$ and $\sigma_{k}$ can only be partly exploited by a retrieval when using HM or AM. The impact of known kinematic variables on the median of $d_{f}$ and the relative uncertainty are presented in Figs. 6 and 7, respectively. When using LM, a significant increase of $d_{f}$ can be found only for $N_{0}^{*}$ and $c$. The increase of the latter is, however, compensated by the more negative $d_{f}$ values for $d$ so that for both quantities together only a small net effect remains. This leads to a reduction of the posterior uncertainty of $\log _{10} c$ by 10 percentage points. For AM, $d_{f}$ is increased for all variables but $D_{m}$ - where $d_{f}$ is already large-and $d$, which is compensated by the increase of $c$. The reduction of posterior uncertainty is particularly strong for $b$, and - when using more than one frequency $-\alpha$. When assuming a smaller $\mathbf{S}_{b}$, the uncertainties for $\alpha$ and $b$ can be further reduced (not shown). Note that when using only HM the impact of prior knowledge about kinematics is less, which is likely related to the fact that $W$ is not included in HM.

\section{b. Radar calibration}

Radar calibration is still an urgent topic, and comparisons with satellite-based radar revealed large offsets (Protat et al. 2011). Of the radar moments, only $Z_{e}$ is 
affected by radar calibration (exceptions are possible for very low SNR ratios). The slopes are only a relative measure between the noise level and the top of the peak, and they should not be affected by calibration either. However, correct determination of the noise level is crucial for estimating the slopes correctly from the simulated Doppler spectrum. Because the absolute estimation of the noise level depends on the calibration as well, the slopes are indirectly affected by calibration offsets, a fact that is also considered in the following. To investigate the impact of a potential radar miscalibration on retrieval bias, the retrieval was applied to synthetic observations assuming a relatively large calibration offset of $\pm 3 \mathrm{~dB}$. A comparison with the retrieval configured without calibration offset revealed only a small increase of the bias of $1 \%-5 \%$ for most variables (not shown). Only for $w$ and $\alpha$, the change in bias is found to be up to $10 \%$ for some retrieval configurations. For all variables, the median bias is still within the 25th-75th-percentile range of the spread among the ISDAC profiles.

\section{c. Increased forward-model error}

To investigate the impact of a doubled forward-model and measurement error, $\mathbf{S}_{e}$ has been multiplied by a factor of 4 (section 2h). Figure 6 shows how this reduces $d_{f}$ by up to 0.2 . The reduction is particularly strong for $\mu^{*}, d$, and $\sigma_{k}$ with AM but is negligible for all quantities when using $\mathrm{LM}$ as well as for $\alpha, c$, and $w$ with AM. A reduced $d_{f}$ value leads to an increased posteriori uncertainty, but for AM and all quantities except $\sigma_{k}$ and $D_{m}$, the median stays between the 25 th and 75 th percentiles of the normal retrieval configuration (Fig. 7). Increasing $\mathbf{S}_{e}$ has negligible impact on the bias of the retrieval solution (not shown).

\section{Discussion and outlook}

The potential of adding higher-order moments of the radar Doppler spectrum (Doppler spectrum width $\sigma$, skewness $\gamma$, and kurtosis $\kappa$ ) as well as the left and right slope of the Doppler spectrum ( $S_{l}$ and $S_{r}$, respectively) to a retrieval targeted to Arctic ice clouds with low to medium turbulence conditions was investigated with a vertically pointing radar. An optimal-estimation-based retrieval was set up that retrieves quantities describing microphysical properties such as $N(D)\left(N_{0}^{*}, D_{m}\right.$, and $\left.\mu^{*}\right)$, $m(D)(\alpha$ and $b)$, and $A(D)(c$ and $d)$ as well as kinematic quantities describing air motion and turbulence ( $w$ and $\left.\sigma_{k}\right)$. To the authors' knowledge, this is the first study to characterize microphysical quantities including ice particle mass (and indirectly density) at the same time as kinematic properties based on radar observations and the first study using higher-order moments and slopes for ice-cloud retrievals. Both real and synthetic radar observations obtained during the ISDAC campaign around Barrow were used. The use of a single, homogeneous dataset allowed for a consistent definition of particle dimension.

The key findings of this study can be summarized as follows:

1) Higher-order moments and slopes respond to quantities describing microphysical and kinematic properties even though they are more noisy than lower-order moments.

2) For single-frequency observations, the use of lowerand higher-order moments as well as the slopes (AM) can double the information content in comparison with the use of lower-order moments (LM). When using more than one frequency, the increase in information content is larger for AM than for LM. The information that higher-order moments and slopes can contribute to a retrieval varies from profile to profile.

3) When using only higher-order moments and the slopes (HM), retrieval performance is between those of LM and AM.

4) The use of AM reduces the uncertainty of all state quantities with respect to the prior knowledge. The use of multiple frequencies can reduce the posterior uncertainties for all quantities even further. Best results can be obtained when using AM and a triple-frequency configuration.

5) Application to real MMCR observations shows only minor differences with synthetic radar observations. This result indicates the general feasibility of the approach.

6) Prior knowledge about kinematic quantities enhances retrieval results-in particular, for $m(D)$-while an increased measurement/forward-model error decreases retrieval results. Calibration offset was found to have only a secondary impact on the results. Doubling of the combined measurement uncertainty and forwardmodel error leads to enhanced retrieval uncertainties but has no impact on the general conclusions.

Together with the methods to estimate the a priori dataset developed in Maahn et al. (2015), this study introduces a generalized retrieval framework that can also be transferred to periods other than ISDAC and/or locations other than Barrow. The question of whether this is also true for the a priori dataset itself has yet to be answered. For this, the representativeness of the used ISDAC dataset has to be investigated. This could be achieved by, for example, comparing the prior information of microphysical properties with other studies (e.g., Wood et al. 2014). Because the aircraft was mostly 
probing above cloud base, it also has to be estimated as to whether the prior can be successfully applied to snowfall consisting of larger particles. As of now, the applicability of the retrieval method is limited to ice clouds consisting of aggregates. To overcome this limitation, the applicability of SSRG theory to heavily rimed and pristine particles has to be investigated. Moreover, a key assumption in the retrieval is that knowledge of $D$, $m$, and aspect ratio is sufficient to estimate the ensemble backscattering properties (Hogan and Westbrook 2014). If this assumption is invalid (e.g., because of an additional dependence on particle type), the retrieval results might be biased to a degree that exceeds the reported uncertainties. The same is true for the estimation of fall velocity, which was assumed to depend only on $D, m$, and $A$ (Heymsfield and Westbrook 2010).

Most Arctic clouds are mixed phase (Battaglia and Delanoë 2013), and the retrieval includes no limitations with respect to lightly rimed particles. However, the retrieval is not designed for mixed-phase clouds for which the SCLW is visible in the radar Doppler spectrum. Therefore, a cloud-classification algorithm (e.g., Shupe 2007) has to be used when applying the retrieval in mixed-phase clouds. An extension of the retrieval to mixed-phase clouds is desirable but requires the addition of multiple, additional variables to the state vector. Moreover, high amounts of liquid can create bimodal peaks or even two separate peaks in the radar Doppler spectrum (Luke et al. 2010). For these peaks, new moments or other quantities have to be developed to express the bimodality properly. As an alternative, the full Doppler spectrum could be used instead of radar moments and slopes for the observation vector $\mathbf{y}$ [as was done for rain by Tridon and Battaglia (2015)]. Using the full spectrum would also allow one to investigate whether there is additional information included in Doppler spectra of ice clouds that cannot be obtained from moments and slopes. Another possibility would be the use of radar attenuation, which is stronger for liquid water (Gosset and Sauvageot 1992), or the inclusion of other remote sensing instruments such as microwave radiometer and lidar that are sensitive to cloud water.

Even though the statistical agreement of retrieval results of real and synthetic radar observations indicates that the retrieval gives consistent results for real observations as well, its output has to be evaluated. This is challenging because collocated, independent observations of microphysical properties do not exist. Possible options would be comparison with models such as numerical weather models or large-eddy simulations (e.g., Solomon et al. 2014), comparison with surface observations (e.g., Wood et al. 2014), or radiative closure studies (e.g., Shupe et al. 2015).
A Monte Carlo-based retrieval approach could be used as an alternative to optimal estimation. Even though it is much more expensive, it could be used for selected profiles to map the solution space and to investigate whether optimal estimation converges to a global minimum or to a local minimum (Posselt and Mace 2014).

Estimating the scattering properties from databasesupported discrete dipole approximation calculations (e.g., Liu 2008; Tyynelä et al. 2011) is potentially more accurate than the self-similar Rayleigh-Gans approximation and could increase the quality of the retrieval. The problem has yet to be solved, however, as to how particle distributions that follow arbitrary mass-size and area-size relations (as it is required by retrievals) can be obtained from a database that contains only certain particle types with certain discrete mass-size and areasize relations. One possibility to solve this issue would be to select different particles from the database and mix them such that the mixture matches the required microphysical bulk properties.

Acknowledgments. The authors thank Robert C. Jackson, Stefan Kneifel, Pavlos Kollias, Edward P. Luke, and Greg M. McFarquhar for providing data and fruitful discussions. This study was carried out within the project ADMIRARI II supported by the German Research Association (DFG) under research Grant LO901/ 5-1 and was also supported by Energy Transition and Climate Change (ET-CC) under DFG Grant ZUK 81. Author M. Maahn was also supported by Grant GSGS2015B-F03 of the Graduate School of Geosciences of the University of Cologne. Data were obtained from the Atmospheric Radiation Measurement Program sponsored by the U.S. Department of Energy Office of Science Office of Biological and Environmental Research (BER) Climate and Environmental Sciences Division. Computing time was gratefully allocated by the Cologne High Efficiency Operating Platform for Sciences (CHEOPS) at the University of Cologne. We thank the editor and the three anonymous reviewers for their helpful comments.

\section{REFERENCES}

Battaglia, A., and J. Delanoë, 2013: Synergies and complementarities of CloudSat-CALIPSO snow observations. J. Geophys. Res. Atmos., 118, 721-731, doi:10.1029/2012JD018092.

Bharadwaj, N., and K. Johnson, 2003: Millimeter wavelength cloud radar (MMCRSPECMOM); $71.323^{\circ} \mathrm{N}, 156.609^{\circ} \mathrm{W}$, North Slope Alaska (NSA) Central Facility, Barrow Alaska (C1). Subset used: 1-30 April 2008, Atmospheric Radiation Measurement Program Climate Research Facility Data Archive, accessed 4 May 2015, doi:10.5439/1025241.

Brown, P. R. A., and P. N. Francis, 1995: Improved measurements of the ice water content in cirrus using a total-water probe. J. Atmos. Oceanic Technol., 12,410-414, doi:10.1175/ 1520-0426(1995)012<0410:IMOTIW>2.0.CO;2. 
Cadeddu, M., 1993: Microwave radiometer (MWRLOS); $71.323^{\circ} \mathrm{N}$, $156.609^{\circ} \mathrm{W}$, North Slope Alaska (NSA) Central Facility, Barrow Alaska (C1). Subset used: 1-30 April 2008, Atmospheric Radiation Measurement Program Climate Research Facility Data Archive, accessed 23 July 2012, doi:10.5439/1046211.

Delanoë, J., and R. J. Hogan, 2008: A variational scheme for retrieving ice cloud properties from combined radar, lidar, and infrared radiometer. J. Geophys. Res., 113, D07204, doi:10.1029/2007JD009000.

—, A. Protat, J. Testud, D. Bouniol, A. J. Heymsfield, A. Bansemer, P. R. A. Brown, and R. M. Forbes, 2005: Statistical properties of the normalized ice particle size distribution. J. Geophys. Res., 110, D10201, doi:10.1029/2004JD005405.

Deng, M., and G. G. Mace, 2006: Cirrus microphysical properties and air motion statistics using cloud radar Doppler moments. Part I: Algorithm description. J. Appl. Meteor. Climatol., 45, 1690-1709, doi:10.1175/JAM2433.1.

Ebell, K., U. Löhnert, S. Crewell, and D. D. Turner, 2010: On characterizing the error in a remotely sensed liquid water content profile. Atmos. Res., 98, 57-68, doi:10.1016/ j.atmosres.2010.06.002.

Gosset, M., and H. Sauvageot, 1992: A dual-wavelength radar method for ice-water characterization in mixed-phase clouds. J. Atmos. Oceanic Technol., 9, 538-547, doi:10.1175/ 1520-0426(1992)009<0538:ADWRMF > 2.0.CO;2.

Grecu, M., and W. S. Olson, 2008: Precipitating snow retrievals from combined airborne cloud radar and millimeter-wave radiometer observations. J. Appl. Meteor. Climatol., 47, 16341650, doi:10.1175/2007JAMC1728.1.

Gultepe, I., A. J. Heymsfield, and D. H. Lenschow, 1990: A comparison of vertical velocity in cirrus obtained from aircraft and lidar divergence measurements during FIRE. J. Atmos. Oceanic Technol., 7, 58-67, doi:10.1175/1520-0426(1990)007<0058: ACOVVI $>2.0 . \mathrm{CO} ; 2$.

Heymsfield, A. J., and C. D. Westbrook, 2010: Advances in the estimation of ice particle fall speeds using laboratory and field measurements. J. Atmos. Sci., 67, 2469-2482, doi:10.1175/ 2010JAS3379.1.

— C. Schmitt, A. Bansemer, and C. H. Twohy, 2010: Improved representation of ice particle masses based on observations in natural clouds. J. Atmos. Sci., 67, 3303-3318, doi:10.1175/ 2010JAS3507.1.

Hogan, R. J., and C. D. Westbrook, 2014: Equation for the microwave backscatter cross section of aggregate snowflakes using the self-similar Rayleigh-Gans approximation. J. Atmos. Sci., 71, 3292-3301, doi:10.1175/JAS-D-13-0347.1.

— A. J. Illingworth, and H. Sauvageot, 2000: Measuring crystal size in cirrus using 35- and 94-GHz radars. J. Atmos. Oceanic Technol., 17, 27-37, doi:10.1175/1520-0426(2000)017<0027: MCSICU>2.0.CO;2.

—, L. Tian, P. R. A. Brown, C. D. Westbrook, A. J. Heymsfield, and J. D. Eastment, 2012: Radar scattering from ice aggregates using the horizontally aligned oblate spheroid approximation. J. Appl. Meteor. Climatol., 51, 655-671, doi:10.1175/ JAMC-D-11-074.1.

Intrieri, J. M., G. L. Stephens, W. L. Eberhard, and T. Uttal, 1993: A method for determining cirrus cloud particle sizes using lidar and radar backscatter technique. J. Appl. Meteor., 32, 1074-1082, doi:10.1175/1520-0450(1993)032<1074:AMFDCC >2.0.CO;2.

Jackson, R. C., and Coauthors, 2012: The dependence of ice microphysics on aerosol concentration in Arctic mixed-phase stratus clouds during ISDAC and M-PACE. J. Geophys. Res., 117, D15207, doi:10.1029/2012JD017668.
Kalesse, H., and P. Kollias, 2013: Climatology of high cloud dynamics using profiling ARM Doppler radar observations. J. Climate, 26, 6340-6359, doi:10.1175/JCLI-D-12-00695.1.

Kneifel, S., M. S. Kulie, and R. Bennartz, 2011: A triple-frequency approach to retrieve microphysical snowfall parameters. J. Geophys. Res., 116, D11203, doi:10.1029/2010JD015430.

—, A. von Lerber, J. Tiira, D. Moisseev, P. Kollias, and J. Leinonen, 2015: Observed relations between snowfall microphysics and triple-frequency radar measurements. J. Geophys. Res. Atmos., 120, 6034-6055, doi:10.1002/ 2015JD023156.

_ , P. Kollias, A. Battaglia, J. Leinonen, M. Maahn, H. Kalesse, and F. Tridon, 2016: First observations of triple-frequency radar Doppler spectra in snowfall: Interpretation and applications. Geophys. Res. Lett., 43, 2225-2233, doi:10.1002/ 2015GL067618.

Kollias, P., E. E. Clothiaux, M. A. Miller, E. P. Luke, K. L. Johnson, K. P. Moran, K. B. Widener, and B. A. Albrecht, 2007: The Atmospheric Radiation Measurement Program cloud profiling radars: Second-generation sampling strategies, processing, and cloud data products. J. Atmos. Oceanic Technol., 24, 1199-1214, doi:10.1175/JTECH2033.1.

, J. Rémillard, E. Luke, and W. Szyrmer, 2011: Cloud radar Doppler spectra in drizzling stratiform clouds: 1. Forward modeling and remote sensing applications. J. Geophys. Res., 116, D13201, doi:10.1029/2010JD015237.

Leinonen, J., and D. Moisseev, 2015: What do triple-frequency radar signatures reveal about aggregate snowflakes? J. Geophys. Res. Atmos., 120, 229-239, doi:10.1002/2014JD022072.

Liu, G., 2008: A database of microwave single-scattering properties for nonspherical ice particles. Bull. Amer. Meteor. Soc., 89, 1563-1570, doi:10.1175/2008BAMS2486.1.

Löhnert, U., S. Crewell, and C. Simmer, 2004: An integrated approach toward retrieving physically consistent profiles of temperature, humidity, and cloud liquid water. J. Appl. Meteor., 43, 1295-1307, doi:10.1175/1520-0450(2004)043<1295: AIATRP $>2.0 . \mathrm{CO} ; 2$.

Luke, E. P., P. Kollias, and M. D. Shupe, 2010: Detection of supercooled liquid in mixed-phase clouds using radar Doppler spectra. J. Geophys. Res., 115, D19201, doi:10.1029/ 2009JD012884.

Maahn, M., 2015: Exploiting vertically pointing Doppler radar for advancing snow and ice cloud observations. Ph.D. dissertation, University of Cologne, 181 pp. [Available online at http://kups. ub.uni-koeln.de/6002/1/thesis_mmaahn_pub.pdf.]

— measurements using Doppler spectra post-processing. Atmos. Meas. Tech., 5, 2661-2673, doi:10.5194/amt-5-2661-2012.

—, U. Löhnert, P. Kollias, R. C. Jackson, and G. M. McFarquhar, 2015: Developing and evaluating ice cloud parameterizations for forward modeling of radar moments using in situ aircraft observations. J. Atmos. Oceanic Technol., 32, 880-903, doi:10.1175/JTECH-D-14-00112.1.

Mace, G. G., A. J. Heymsfield, and M. R. Poellot, 2002: On retrieving the microphysical properties of cirrus clouds using the moments of the millimeter-wavelength Doppler spectrum. J. Geophys. Res., 107, 4815, doi:10.1029/2001JD001308.

Matrosov, S. Y., 2007: Modeling backscatter properties of snowfall at millimeter wavelengths. J. Atmos. Sci., 64, 1727-1736, doi:10.1175/JAS3904.1.

2011: Feasibility of using radar differential Doppler velocity and dual-frequency ratio for sizing particles in thick ice clouds. J. Geophys. Res., 116, D17202, doi:10.1029/2011JD015857. 
— A. V. Korolev, and A. J. Heymsfield, 2002: Profiling cloud ice mass and particle characteristic size from Doppler radar measurements. J. Atmos. Oceanic Technol., 19, 1003-1018, doi:10.1175/1520-0426(2002)019<1003:PCIMAP>2.0.CO;2.

McFarquhar, G., and R. Jackson, 2012: ISDAC microphysics; NRC Convair-580. Subset used: 1-30 April 2008, Atmospheric Radiation Measurement Program Climate Research Facility Data Archive, accessed 20 March 2014, doi:10.5439/1171942.

-_, and Coauthors, 2011: Indirect and Semi-Direct Aerosol Campaign: The impact of Arctic aerosols on clouds. Bull. Amer. Meteor. Soc., 92, 183-201, doi:10.1175/ 2010BAMS2935.1.

Mishchenko, M. I., 2000: Calculation of the amplitude matrix for a nonspherical particle in a fixed orientation. Appl. Opt., 39, 1026-1031, doi:10.1364/AO.39.001026.

Moninger, W. R., S. G. Benjamin, B. D. Jamison, T. W. Schlatter, T. L. Smith, and E. J. Szoke, 2010: Evaluation of regional aircraft observations using TAMDAR. Wea. Forecasting, 25, 627-645, doi:10.1175/2009WAF2222321.1.

Moran, K. P., B. E. Martner, M. J. Post, R. A. Kropfli, D. C. Welsh, and K. B. Widener, 1998: An unattended cloud-profiling radar for use in climate research. Bull. Amer. Meteor. Soc., 79, 443-455, doi:10.1175/1520-0477(1998)079<0443:AUCPRF>2.0.CO;2.

Nemarich, J., R. J. Wellman, and J. Lacombe, 1988: Backscatter and attenuation by falling snow and rain at 96, 140, and 225 GHz. IEEE Trans. Geosci. Remote Sens., 26, 319-329, doi:10.1109/36.3034.

Posselt, D. J., and G. G. Mace, 2014: MCMC-based assessment of the error characteristics of a surface-based combined radarpassive microwave cloud property retrieval. J. Appl. Meteor. Climatol., 53, 2034-2057, doi:10.1175/JAMC-D-13-0237.1.

Protat, A., D. Bouniol, E. J. O'Connor, H. Klein Baltink, J. Verlinde, and K. Widener, 2011: CloudSat as a global radar calibrator. J. Atmos. Oceanic Technol., 28, 445-452, doi:10.1175/ 2010JTECHA1443.1.

Rodgers, C. D., 2000: Inverse Methods for Atmospheric Sounding: Theory and Practice. World Scientific, $256 \mathrm{pp}$.

Sekelsky, S. M., W. L. Ecklund, J. M. Firda, K. S. Gage, and R. E. McIntosh, 1999: Particle size estimation in ice-phase clouds using multifrequency radar reflectivity measurements at 95 , 33, and 2.8 GHz. J. Appl. Meteor., 38, 5-28, doi:10.1175/ 1520-0450(1999)038<0005:PSEIIP > 2.0.CO;2.

Shupe, M. D., 2007: A ground-based multisensor cloud phase classifier. Geophys. Res. Lett., 34, L22809, doi:10.1029/ 2007 GL031008.

- - P. Kollias, M. Poellot, and E. Eloranta, 2008: On deriving vertical air motions from cloud radar Doppler spectra. J. Atmos. Oceanic Technol., 25, 547-557, doi:10.1175/ 2007JTECHA1007.1.

- I. M. Brooks, and G. Canut, 2012: Evaluation of turbulent dissipation rate retrievals from Doppler cloud radar. Atmos. Meas. Tech., 5, 1375-1385, doi:10.5194/amt-5-1375-2012.

— D. D. Turner, A. Zwink, M. M. Thieman, E. J. Mlawer, and T. Shippert, 2015: Deriving Arctic cloud microphysics at Barrow, Alaska: Algorithms, results, and radiative closure. J. Appl. Meteor. Climatol., 54, 1675-1689, doi:10.1175/ JAMC-D-15-0054.1.
Smith, P. L., 2010: The unit symbol for the logarithmic scale of radar reflectivity factors. J. Atmos. Oceanic Technol., 27, 615616, doi:10.1175/2009JTECHA1360.1.

Solomon, A., M. D. Shupe, O. Persson, H. Morrison, T. Yamaguchi, P. M. Caldwell, and G. de Boer, 2014: The sensitivity of springtime Arctic mixed-phase stratocumulus clouds to surfacelayer and cloud-top inversion-layer moisture sources. J. Atmos. Sci., 71, 574-595, doi:10.1175/JAS-D-13-0179.1.

Steinke, S., U. Löhnert, S. Crewell, and S. Liu, 2014: Water vapor tomography with two microwave radiometers. IEEE Trans. Geosci. Remote Sens., 11, 419-423, doi:10.1109/LGRS.2013.2264354.

Szyrmer, W., and I. Zawadzki, 2014: Snow studies. Part IV: Ensemble retrieval of snow microphysics from dual-wavelength vertically pointing radars. J. Atmos. Sci., 71, 1171-1186, doi:10.1175/JAS-D-12-0286.1.

_ A. Tatarevic, and P. Kollias, 2012: Ice clouds microphysical retrieval using 94-GHz Doppler radar observations: Basic relations within the retrieval framework. J. Geophys. Res., 117, D14203, doi:10.1029/2011JD016675.

Testud, J., S. Oury, R. A. Black, P. Amayenc, and X. Dou, 2001: The concept of "normalized" distribution to describe raindrop spectra: A tool for cloud physics and cloud remote sensing. J. Appl. Meteor., 40, 1118-1140, doi:10.1175/ 1520-0450(2001)040<1118:TCONDT > 2.0.CO;2.

Tridon, F., and A. Battaglia, 2015: Dual-frequency radar Doppler spectral retrieval of rain drop size distributions and entangled dynamics variables. J. Geophys. Res. Atmos., 5585-5601, doi:10.1002/2014JD023023.

Turner, D. D., and U. Löhnert, 2014: Information content and uncertainties in thermodynamic profiles and liquid cloud properties retrieved from the ground-based Atmospheric Emitted Radiance Interferometer (AERI). J. Appl. Meteor. Climatol., 53, 752-771, doi:10.1175/JAMC-D-13-0126.1.

Tyynelä, J., J. Leinonen, D. Moisseev, and T. Nousiainen, 2011: Radar backscattering from snowflakes: Comparison of fractal, aggregate, and soft spheroid models. J. Atmos. Oceanic Technol., 28, 1365-1372, doi:10.1175/JTECH-D-11-00004.1.

Verlinde, J., M. P. Rambukkange, E. E. Clothiaux, G. M. McFarquhar, and E. W. Eloranta, 2013: Arctic multilayered, mixed-phase cloud processes revealed in millimeter-wave cloud radar Doppler spectra. J. Geophys. Res. Atmos., 118, 13 199-13 213, doi:10.1002/2013JD020183.

Warren, S. G., and R. E. Brandt, 2008: Optical constants of ice from the ultraviolet to the microwave: A revised compilation. J. Geophys. Res., 113, D14220, doi:10.1029/2007JD009744.

Widener, K. B., and K. Johnson, 2005: Millimeter Wave Cloud Radar handbook. Dept. of Energy Office of Science ARM Program Tech. Rep. ARM TR-018, 22 pp. [Available online at https://www.arm.gov/publications/tech_reports/handbooks/ mmcr_handbook.pdf.]

Wood, N. B., T. S. L'Ecuyer, A. J. Heymsfield, G. L. Stephens, D. R. Hudak, and P. Rodriguez, 2014: Estimating snow microphysical properties using collocated multisensor observations. J. Geophys. Res. Atmos., 119, 8941-8961, doi:10.1002/2013JD021303.

Zrnić, D. S., 1975: Simulation of weatherlike Doppler spectra and signals. J. Appl. Meteor., 14, 619-620, doi:10.1175/ 1520-0450(1975)014<0619:SOWDSA > 2.0.CO;2. 